LLNL-JRNL-697380-DRAFT

Preprint typeset using LATEX style emulateapj v. 8/13/10

\title{
PROBABILISTIC COSMOLOGICAL MASS MAPPING FROM WEAK LENSING SHEAR
}

\author{
M. D. Schneider ${ }^{1}$, K. Y. NG ${ }^{2,3}$, W. A. Dawson ${ }^{1}$, P. J. Marshall ${ }^{4,5}$, J. Meyers ${ }^{4}$, D. J. Bard ${ }^{3}$ \\ (Dated: Draft October 24, 2016) \\ LLNL-JRNL-697380-DRAFT
}

\begin{abstract}
We infer gravitational lensing shear and convergence fields from galaxy ellipticity catalogs under a spatial process prior for the lensing potential. We demonstrate the performance of our algorithm with simulated Gaussian-distributed cosmological lensing shear maps and a reconstruction of the mass distribution of the merging galaxy cluster Abell 781 using galaxy ellipticities measured with the Deep Lens Survey. Given interim posterior samples of lensing shear or convergence fields on the sky, we describe an algorithm to infer cosmological parameters via lens field marginalization. In the most general formulation of our algorithm we make no assumptions about weak shear or Gaussian distributed shape noise or shears. Because we require solutions and matrix determinants of a linear system of dimension that scales with the number of galaxies, we expect our algorithm to require parallel high-performance computing resources for application to ongoing wide field lensing surveys. Subject headings: gravitational lensing: weak; methods: data analysis; methods: statistical; catalogs; surveys; cosmology: observations
\end{abstract}

\section{INTRODUCTION}

The state of the inhomogeneous cosmic matter distribution (and the time-dependent dark energy density) can be measured by the gravitational lensing shearing and magnification of background luminous sources. Using galaxies as the back lights for gravitational lensing ("cosmic shear") allows tomographic reconstruction of the matter distribution along the line of sight. But, inference of galaxy lensing shears is clouded by the unknown distribution (and possibly correlation) of galaxy intrinsic morphologies as well as image systematics that induce spurious ellipticity correlations.

Common inference algorithms for gravitational lensing shear involve cross-correlating the ellipticities of galaxies in a two-point function estimator under the assumption of calibrated intrinsic ellipticity distributions and alignments (e.g., Jee et al. 2016). This is a lossy procedure because the angular phase information in the lensing shear and magnification fields on the sky is discarded. Traditional algorithms are also necessarily biased because of the need to calibrate the unknown galaxy ellipticity distributions. Said another way, the two-point function summary statistics of cosmological large-scale structure do not capture the full statistical information in the multivariate distribution of the lensing observables(e.g., Pan \& Szapudi 2005; Hamilton et al. 2006; Takada \& Hu 2013; Carron 2014; Petri et al. 2016)

In this paper we revisit the problem of inferring the lensing convergence posterior distribution from a catalog of galaxy ellipticities to potentially capture more cosmo-

\footnotetext{
${ }^{1}$ Lawrence Livermore National Laboratory, Livermore, CA 94551, USA.

${ }^{2}$ University of California, Davis, Davis, CA 95616, USA.

${ }^{3}$ National Energy Research Scientific Computing Center, Lawrence Berkeley National Laboratory, 1 Cyclotron Road, Berkeley, CA 94720-8150, USA.

${ }^{4}$ Kavli Institute for Particle Astrophysics and Cosmology, Stanford University, Stanford, CA 94035, USA.

5 SLAC National Accelerator Laboratory, Menlo Park, CA 94025, USA.
}

logical information from cosmic shear than is available in two-point function estimators of the shear. We pursue a probabilistic approach to lensing convergence inference as a means to propagate the large uncertainties that can come in the presence of galaxy shape noise, finite survey areas, masking, and sample selection. Moreover, we aim to build an inference framework that can fit within our previous work on probabilistic cosmic shear (Schneider et al. 2015).

Tyson et al. (1990) first presented a method to reconstruct the projected mass distribution from weak gravitational lensing shears of source galaxy images. Kaiser \& Squires (1993) derived the lensing convergence in terms of the shear (a nonlocal relation) and applied this theory to estimators of the convergence given a measured galaxy ellipticity catalog. The method of Kaiser \& Squires (1993), while theoretically sound, requires unbroken sky coverage and low-pass filtering to yield finite noise in the lensing convergence estimator. The Kaiser \& Squires (1993) method remains useful, however, for visualization purposes (Chang et al. 2015; Vikram et al. 2015). Subsequent papers have extended, applied, and explored the limitations of filtering algorithms for cluster mass mapping (Bartelmann 1995; Schneider \& Seitz 1995; Lombardi \& Bertin 1998; Gray et al. 2002; Dietrich et al. 2005; Massey et al. 2007; Deriaz et al. 2012; Amara et al. 2012; Van Waerbeke et al. 2013). Remaining data analysis challenges using such methods include finite survey boundaries and masks, separation of $\mathrm{E}$ and $\mathrm{B}$ modes in the shear field, noise or significance characterization for shear or convergence extrema, and the requirement in many algorithms to smooth or average the ellipticities of galaxies before the convergence inference process.

Maximum likelihood (ML) estimators for the lensing convergence (e.g., Bartelmann et al. 1996; Seitz et al. 1998; Heymans et al. 2008; Kubo et al. 2009) can help mitigate biases arising from survey masks and admit mathematically consistent noise characterization (van Waerbeke 2000). However such estimators are biased. And many approaches often still require a preliminary 
smoothing of the observed galaxy ellipticity field.

Bridle et al. (1998) and Bridle et al. (2000) introduced a 'maximum entropy' Bayesian prior for the lensing convergence from information theoretic and Bayesian analysis perspectives to derive an estimator for the projected mass distribution of galaxy clusters with desirable noise properties in a finite field. Marshall et al. (2001) and Starck et al. (2001) refined the maximum entropy method to specify the optimal smoothing length scale for galaxy cluster mass inference via the Bayesian evidence of the observed ellipticities. Marshall et al. (2013) released a code $^{6}$ implementing the algorithm from Marshall et al. (2001). The algorithm of Marshall et al. (2001) is close to meeting all the requirements for the current analysis, except the choice of smoothing scale and application to field rather than cluster lensing is not demonstrated in the literature. We will show further benefits of the algorithm developed in this paper below. Starck et al. (2006) applied a modification of the maximum entropy method to a cosmic shear analysis using $N$-body cosmological simulations to create mock observations. The work of Starck et al. (2006) and also Jiao et al. (2011) include maximum entropy algorithm modifications to better handle masking, shape noise, and degrees of smoothing.

For constraining cosmological parameters, several groups have considered the abundance of peaks in lensing convergence maps (Kratochvil et al. 2010; Schmidt \& Rozo 2011; Shan et al. 2014; Liu et al. 2015; Reischke et al. 2016; Liu \& Haiman 2016; Liu et al. 2016; Petri et al. 2016). These studies have a common approach in direct calculation or estimation of lensing peaks without an attempt to infer associated peaks in the 3D cosmological mass density. The measurement process is thus direct as lensing by multiple structures or voids along any given line of sight can easily confuse the 2D to 3D mass inference. Just as the abundance of galaxy clusters provides tight constraints on the cosmological model, so too can the abundance of lensing peaks. However, the estimators for lensing peaks often involve averages or line integrals over contiguous sky areas, which are confounded by survey masks (Liu et al. 2014). The bias introduced by masking may be overcome with forward simulations of the nonlinear lensing convergence field, which has been achieved with $N$-body cosmological simulations of largescale structure combined with ray-tracing predictions of the lensing statistics (e.g., Bard et al. 2016; Petri et al. 2016).

In a complimentary approach to cosmological parameter inference, other groups have considered a linear theory approximation to (suitably smoothed) lensing mass maps. Specifically, if (i) the shear field can be approximated as Gaussian distributed, (ii) the noise distribution in the galaxy ellipticity measurements (and intrinsic shape distribution) can also be approximated as Gaussian distributed, and (iii) the weak shear approximation is valid, then mass maps can be obtained using the Wiener Filter (WF) (Wiener 1949). The WF is a wellstudied signal-inference algorithm that has been applied to cosmic microwave background (CMB) analysis with great success in a Bayesian context (Wandelt et al. 2004). Alsing et al. (2015) and Alsing et al. (2016) have recently

6 LENSENT2, http://www.slac.stanford.edu/ pjm/lensent/ version2/index.html extended the WF technology from the CMB literature to the problem of weak lensing shear field and power spectrum inference. However, these WF approaches are limited not only by the WF assumptions above but also by requirements to smooth the measured galaxy ellipticity field to a uniform grid on the sky and to work solely within a linear theory approximation for the distribution of cosmological mass density perturbations. Working within the stated assumptions, the primary challenge for WF approaches is the computation of large matrix solve operations. Novel and effective algorithms for sampling from the WF distribution have been demonstrated for the CMB (Elsner \& Wandelt 2013; Racine et al. 2016) that are also effective for smoothed, weak-shear, and linear theory cosmic shear inferences (Alsing et al. 2016).

This paper is structured as follows. In Section 2 we describe the statistical framework for lensing convergence and shear inference given galaxy images or a galaxy ellipticity catalog. In subsection 2.1 we describe the Gaussian Process (GP) prior for the lensing potential and how this informs the correlated inferences of shear and convergence. We give a prescription for GP parameter optimization in subsubsection 2.1.1. We apply our method to infer lensing convergence maps of simulated ellipticity catalogs in subsection 3.1 and of an observed galaxy cluster in subsection 3.2. We describe our main conclusions in Section 4. We provide details of the GP covariance derivation for lensing shear and convergence fields in Appendix A and provide lensing map inferences under an analogous covariance for a cosmological model with a linear theory approximation in Appendix B.

\section{METHOD}

We describe a joint probabilistic model for the lensing shear and convergence given galaxy imaging data. We previously presented the complete statistical framework for cosmic shear inference (Schneider et al. 2015) and here focus on the specifics of lensing convergence inference as a function of sky coordinates in both linear and nonlinear regimes of the cosmological mass density perturbations. In Figure 1 (left panel) we show the relationships in the probabilistic model for CCD galaxy imaging data for multiple observation epochs, multiple galaxies, and multiple galaxy samples (e.g., samples selected in different photometric redshift bins).

The model for observed galaxy images requires (at least) specification of the point-spread function (PSF), $\Pi_{n_{s}, i}$, at all $n_{s}$ galaxy locations in all epochs $i$, the intrinsic or pre-lensing shape or morphology of the galaxy, $e_{n_{s}}$, the applied lensing shear and magnification, $\Upsilon_{s}$, and the noise properties of the pixelated image, $\sigma_{n_{s}, i}$ (e.g., Gaussian with an asserted r.m.s.).

To simplify the discussion for this paper focused on shear inference, we assume the $\operatorname{PSF} \Pi_{n_{s}, i}$ is known at the locations of all galaxies in all epochs. We do not further consider errors in the PSF inference in this work. Following Schneider et al. (2015) we allow for the intrinsic ellipticity (and potentially size, fluxes, etc.) of each galaxy to be drawn from galaxy-specific distributions with parameters $\alpha_{n_{s}}$. The parameters $\alpha_{n_{s}}$ in turn are hierarchically distributed under a distribution with with parameters $a_{\mathrm{DP}}$, allowing inference of the effective 'shape noise' in the shear inference. We use the label $\Upsilon$ for the model for the lensing shear $\gamma$ and convergence $\kappa$, 

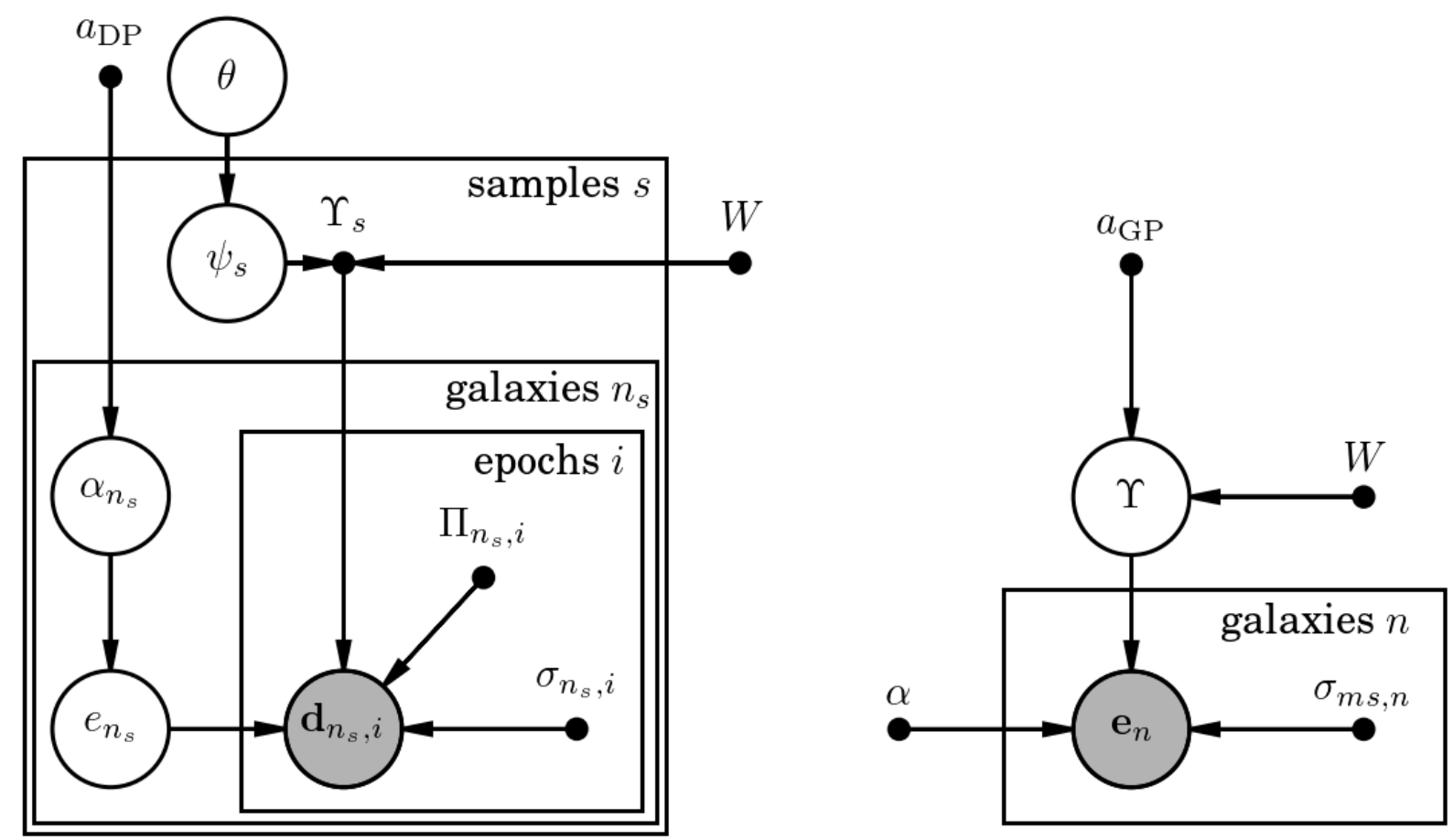

FIG. 1. - Two successive levels of approximation for our statistical model for sampling probabilistic lensing shear and convergence $\Upsilon$ fields. The unshaded circles indicate sampling parameters. Shaded circles indicate observed parameters while dots indicate parameters with fixed values rather than being sampled. Left panel: The model for galaxy image pixel data $\mathbf{d}_{n, i}$ for each galaxy $n$ and observation epoch $i$ requires specification of the pixel noise $\sigma_{n, i}$, the intrinsic (i.e., unlensed) galaxy ellipticity $e_{n}$, the lensing fields $\Upsilon$, and the PSF at each galaxy location in each epoch $\Pi_{n, i}$. The distribution of galaxy intrinsic ellipticities is described by the parameters $\alpha_{n}$, which can specify distinct distributions for each galaxy $n$. In Schneider et al. (2015) we infer marginal constraints on $\alpha_{n}$ under a Dirichlet Process (DP) prior. For this initial study, we assert a Gaussian Process (GP) prior on the lens fields $\Upsilon$ in this paper, with the assumption that the posterior inferences of $\Upsilon$ will be related to a cosmological model in a separate analysis pipeline as we describe in the text. Here we assert a known PSF $\Pi_{n, i}$ at every galaxy location, again deferring the inference and marginalization of PSFs to a separate paper. The inference of the lens fields also depends on our assertion of the survey window function $W$. Right panel: The approximate statistical model when the galaxy imaging pixel data is summarized as a galaxy ellipticity catalog $\left(e_{n}, \sigma_{e, n}\right.$ for $\left.n=1, \ldots, n_{\text {gal }}\right)$ requires specification of the intrinsic ellipticity distribution parameters $\alpha$ (now assumed the same for all galaxies), the ellipticity measurement uncertainties $\sigma_{\mathrm{ms}, \mathrm{n}}$ per galaxy, and the lens fields $\Upsilon$. We now also assert the variance, $\alpha$ of the assumed zero-mean intrinsic ellipticity distribution.

which uniquely specify the components of the trace-free linear distortion matrix under a weak lensing $(\kappa \ll 1)$ approximation of the lens equation (e.g., Bartelmann \& Schneider 2001).

As illustrated by the 'plates' in the left panel of Figure 1, the PSF is unique to each observation epoch but the intrinsic properties of a galaxy image are common across epochs (for each unique galaxy). A key feature in the graphical model of the left panel in Figure 1 is that the lens fields $\Upsilon_{s}$ are common across all galaxy images in all epochs, indicating that the we require a spatially correlated model for the coherent lensing shear and magnification patterns on the sky. Specifying an appropriate correlated model for the lens fields is the main focus of this paper. We allow the lens field models to be distinct for different galaxy samples $s$ in Figure 1 because, e.g., galaxies in different photometric redshift bins will be lensed by partially different foreground mass distributions. Only at the top level of the graphical model do the lens field inferences from different galaxy samples become connected under a cosmological model with parameters $\theta$.

We therefore explore an interim probabilistic model for the lens fields inferred from distinct galaxy samples $s$ such that inferences of $\Upsilon_{s}$ are statistically independent for different $s$. This will allow us to separate compu- tationally expensive components of a cosmic shear inference pipeline, explore multiple cosmological and systematics models for the data, and eventually perform rigorous uncertainty propagation and marginalization of image and intrinsic galaxy nuisance parameters as outlined in Schneider et al. (2015).

In measuring lensing shear of galaxy images, we must marginalize over the intrinsic ellipticities, $\epsilon^{\mathrm{int}}$, of the images. This is often done by averaging the ellipticities of galaxies in neighboring regions of the sky and redshift, where the weights can include the measurement and shape noise models. But, here we perform a marginalization over an explicit intrinsic ellipticity distribution,

$$
\begin{array}{r}
\operatorname{Pr}(\mathbf{d} \mid \Upsilon(\mathbf{x}), \alpha)=\prod_{n=1}^{n_{\text {gal }}} \int d^{m} \epsilon_{n}^{\text {int }} \operatorname{Pr}\left(\mathbf{d}_{n} \mid \epsilon_{n}^{\text {int }}, \Upsilon\left(\mathbf{x}_{n}\right)\right) \\
\times \operatorname{Pr}\left(\epsilon_{n}^{\text {int }} \mid \alpha\right),
\end{array}
$$

where we assume the likelihood functions for each galaxy image $n=1, \ldots, n_{\text {gal }}$ are statistically independent (see Schneider et al. 2015, for more discussion of this assumption). The data vector $\mathbf{d}_{n}$ is composed of either the pixel values contributing to the image of galaxy $n$ or a summary statistic of those pixel values.

As a pedagogical step in the development of our probabilistic lens field model, we will consider an approximate 
TABLE 1

PARAMETERS FOR THE STATISTICAL MODEL.

\begin{tabular}{cl}
\hline Parameter & Description \\
\hline$i$ & index over epochs, or different exposures of a galaxy \\
$n$ & index over galaxies \\
$\Pi_{n, i}$ & Point Spread Function (PSF) for galaxy $n_{s}$ in epoch $i$ \\
$e_{n}$ & Intrinsic (pre-lensing) ellipticity of a galaxy \\
$\Upsilon_{s}$ & lensing shear and convergence that modify a galaxy image \\
$\psi_{s}$ & lensing potential \\
$\theta$ & cosmological parameters \\
$W$ & survey window function \\
$\sigma_{n, i}$ & noise properties in a galaxy image \\
$\alpha_{n}$ & parameters specifying the intrinsic ellipticity distribution for a galaxy \\
$a_{\mathrm{DP}}$ & parameters specifying the distribution over $\alpha_{n}$ \\
$\mathbf{d}_{\mathbf{n}}$ & data vector (measured $e_{1,2}$ for each galaxy $n$ ) \\
$\sigma_{\mathrm{ms} ; n}$ & ellipticity measurement error for galaxy $n$ \\
$\mathbf{x}$ & vector of $2 \mathrm{D}$ spatial locations of galaxies \\
$\epsilon_{n}^{\mathrm{int}}$ & intrinsic galaxy ellipticity for galaxy $n$ \\
$\alpha \equiv \sigma_{e}^{2}$ & parameters of the distribution of galaxy parameters \\
$\mathrm{W}$ & window function for the survey footprint \\
$a_{\mathrm{GP}}$ & parameters of the GP kernel \\
$\lambda_{\mathrm{GP}}$ & precision of the GP kernel (element of $\left.a_{\mathrm{GP}}\right)$ \\
$\ell_{\mathrm{GP}}^{2}$ & squared GP correlation length (element of $a_{\mathrm{GP}}$ ) \\
&
\end{tabular}

likelihood function for summary statistics of the pixel data; namely estimators for the ellipticity of each galaxy image $e \equiv e_{1}+i e_{2}$ along with an associated measurement error per ellipticity component $\sigma_{\mathrm{ms}}$. See the right panel of Figure 1. We will develop the approximate model for ellipticity measurements as a data vector in this paper but advocate for the more complete algorithm of Schneider et al. (2015) for any data analysis because of the known large biases in using ellipticity estimators for cosmic shear (e.g., Refregier et al. 2012; Kacprzak et al. 2012).

Assuming Gaussian distributed ellipticity measurement errors the likelihood function is then,

$$
\operatorname{Pr}\left(\mathbf{d}_{n} \mid e_{n}, \Upsilon\left(\mathbf{x}_{n}\right)\right)=\mathcal{N}_{\hat{e}_{n}}\left(e_{n}, \sigma_{\mathrm{ms} ; n}^{2} \mathbb{1}_{2}\right),
$$

where we explicitly label the data $\mathbf{d}_{n} \equiv \hat{e}_{n}$ as ellipticity estimators, and the distribution is bivariate given the two ellipticity components.

A general likelihood function depends on the observable galaxy properties such as ellipticity, size, and flux, which are modified by lensing from the intrinsic properties described by $\epsilon^{\text {int }}$. So, to evaluate Equation (2), we define the lensed galaxy parameters,

$$
e_{n}\left(\Upsilon\left(\mathbf{x}_{n}\right)\right) \equiv f\left(\epsilon_{n}^{\mathrm{int}}, \Upsilon\left(\mathbf{x}_{n}\right)\right),
$$

where $f(\cdot)$ denotes the function that transforms the intrinsic, unlensed, galaxy ellipticities $\epsilon_{n}^{\text {int }}$ to those in the lensed model $e_{n}$ under the action of the lensing convergence and shear specified by $\Upsilon\left(\mathbf{x}_{n}\right)$ at the galaxy sky location $\mathbf{x}_{n}$. In the weak shear limit defined by $\kappa \ll 1$, Equation (3) reduces to

$$
\tilde{e}_{n}^{\text {weak-shear }}=\epsilon_{n}^{\text {int }}+g\left(\mathbf{x}_{n}\right),
$$

where $g \equiv \gamma /(1-\kappa) \approx \gamma$ is the reduced shear.

For our pedagogy, we specify a Gaussian distribution for the unlensed galaxy properties $\epsilon^{\text {int }}$ to use in evaluating Equation (1),

$$
\operatorname{Pr}\left(\epsilon_{n}^{\mathrm{int}} \mid \alpha, \Upsilon\left(\mathbf{x}_{n}\right)\right)=\mathcal{N}_{\epsilon_{n}^{\text {int }}}\left(0, \sigma_{e}^{2} \mathbb{1}_{2}\right) .
$$

Using the weak shear approximation in Equation (4), we can perform the marginalization integral in Equation (1) analytically,

$$
\begin{aligned}
\operatorname{Pr}(\mathbf{d} \mid \Upsilon(\mathbf{x}), \alpha)= & \prod_{n=1}^{n_{\text {gal }}} \int d^{2} \epsilon_{n}^{\text {int }} \mathcal{N}_{\epsilon^{\text {int }}}\left(\hat{e}_{n}-g\left(\mathbf{x}_{n}\right), \sigma_{\mathrm{ms} ; n}^{2} \mathbb{1}_{2}\right) \\
& \times \mathcal{N}_{\epsilon^{\text {int }}}\left(0, \sigma_{e}^{2} \mathbb{1}_{2}\right) \\
= & \prod_{n=1}^{n_{\text {gal }}} \mathcal{N}_{g\left(\mathbf{x}_{n}\right)}\left(\hat{e}_{n},\left(\sigma_{\mathrm{ms} ; n}^{2}+\sigma_{e}^{2}\right) \mathbb{1}_{2}\right) \\
= & \mathcal{N}_{\mathrm{g}}(\hat{\mathrm{e}}, \mathrm{N})
\end{aligned}
$$

where in the final line we defined the $2 n_{\text {gal }}$-length vector of observed ellipticities ê, the same-length vector of reduced shear components at each galaxy locations $g$, and the diagonal $2 n_{\text {gal }} \times 2 n_{\text {gal }}$ dimensional covariance matrix $\mathrm{N}$ with the $n$th diagonal entry equal to $\sigma_{\mathrm{ms} ; n}^{2}+\sigma_{e}^{2}$. Note the weak shear likelihood does not depend on the lensing convergence $\kappa$ (because the reduced shear is approximated as equal to the non-reduced shear and we ignore lensing magnification effects on the galaxy sizes and fluxes).

\subsection{A maximum entropy prior for lensing fields}

We want an interim prior on the lensing convergence and shear that is not only independent of cosmology but also broadly encompassing of the possible cosmological interpretations and spatially varying systematics contributions. That is, by choosing a functional form of a prior for interpolating shear over the sky and marginalizing statistical uncertainties, we should not restrict the class of physical models that might explain the data. A mathematical version of this sentiment is that we want a maximum entropy prior (Jaynes 1957; Shore \& Johnson 1980) on the lensing fields.

For an assumed mean and (co-)variance, the Gaussian distribution is the maximum entropy distribution (Vasicek 1976). Therefore, because it is principally the second moment of the lensing convergence that we use 
to constrain cosmological models, we choose a Gaussian Process (GP) interim prior for sampling convergence (and shear) fields on the sky given measurements of galaxy image moments.

Gravitational lensing shear is a spin-2 field and is nonlocally related to the lensing convergence, which both present modeling challenges. However, both the lensing convergence and shear can be derived as second derivatives of a scalar valued lensing potential $\psi$. So we impose the GP prior on the potential $\psi$ and derive the related priors on the convergence $\kappa$ and shear $\gamma$ from this starting assertion.

Each of the lensed observables, $\Upsilon(\mathbf{x}) \equiv\left[\kappa, \gamma_{1}, \gamma_{2}\right]$ is related to the lensing potential $\psi$ via derivatives in the form of:

$$
\begin{aligned}
\kappa & =\frac{1}{2}\left(\frac{\partial^{2} \psi}{\partial x_{1}^{2}}+\frac{\partial^{2} \psi}{\partial x_{2}^{2}}\right)=\frac{1}{2}\left(\psi_{, 11}+\psi_{, 22}\right) \\
\gamma_{1} & =\frac{1}{2}\left(\frac{\partial^{2} \psi}{\partial x_{1}^{2}}-\frac{\partial^{2} \psi}{\partial x_{2}^{2}}\right)=\frac{1}{2}\left(\psi_{, 11}-\psi_{, 22}\right) \\
\gamma_{2} & =\frac{1}{2}\left(\frac{\partial^{2} \psi}{\partial x_{1} \partial x_{2}}+\frac{\partial^{2} \psi}{\partial x_{2} \partial x_{1}}\right)=\frac{1}{2}\left(\psi_{, 12}+\psi_{, 21}\right) .
\end{aligned}
$$

It is straightforward to derive via integration by parts on the moments of the field that if $\psi(\mathbf{x})$ is Gaussian distributed for given $\mathbf{x}$, then so too is $\Upsilon(\mathbf{x})$, but with a modified covariance. By specifying a GP prior on the lens potential, we therefore can derive a GP prior on the combination of lensing convergence and shear fields that preserves the physical correlations between these fields. We will show that we can infer the lensing convergence and shear via correlated draws from a GP distribution given a galaxy ellipticity catalog. We do not then compute lensing convergence and shear from spatial derivatives of a GP-distributed lens potential. The latter operation is only precisely defined when we have (in principle) knowledge of the lens potential at all sky locations. However, galaxies provide a non-contiguous background for measuring the lens potential. The relations in Equation (9), Equation (10), and Equation (11) are then imposed only by the GP covariance structure during sampling and marginalization.

The derivations of using a GP to represent the lensing convergence and shear fields were first presented in $\mathrm{Ng}$ (2016). We refer readers to $\mathrm{Ng}$ (2016) for an introduction to the basics of a GP. We show the derivations from $\mathrm{Ng}$ (2016) in Appendix A for the convenience of the reader. In particular, the GP prior for $\Upsilon$ derived from that for $\psi$ should not mix $\mathrm{E}$ and $\mathrm{B}$ modes in the two-point function because we only allow for GP realizations that preserve the combinations of fields that satisfy Equations (9)-(11) in the two-point correlations. It is also straightforward to extend the scalar valued potential $\psi$ to a complex-valued potential $\psi \rightarrow \psi_{E}+i \psi_{B}$ to model or infer both E and B mode contributions to a measured shear signal.

We choose a squared exponential kernel for the GP model of the lensing potential $\psi$,

$$
\mathrm{S}_{\psi}\left(\mathbf{x}, \mathbf{y} ; \lambda_{\mathrm{GP}}, \ell_{\mathrm{GP}}^{2}\right)=\lambda_{\mathrm{GP}}^{-1} \exp \left(-\frac{1}{2} \frac{s^{2}(\mathbf{x}, \mathbf{y})}{\ell_{\mathrm{GP}}^{2}}\right),
$$

where $\lambda_{\mathrm{GP}}$ is a precision parameter that sets the amplitude of fluctuations of the GP, $\ell_{\mathrm{GP}}^{2}$ is a squared distance defining the correlation length of the GP kernel, and the squared distance $s^{2}$ between pairs of galaxy locations is,

$$
s^{2} \equiv(\mathbf{x}-\mathbf{y})^{T}(\mathbf{x}-\mathbf{y}) .
$$

The squared exponential kernel is useful in interpolating smooth response functions between the observed galaxy locations, as we expect for low resolution or low signalto-noise ratio (SNR) weak lensing mass reconstructions. Exploration of other kernel choices for different mass reconstruction resolutions and SNRs is an interesting question that we leave for later work. A practical motivation for our kernel choice is that the two parameters of the squared exponential kernel can be optimized for different data sets without particular numerical or computational challenges. A further justification for our smooth kernel choice comes from our intention to use the GP as merely an interim prior for sampling lens fields. We will later describe how these interim lens field realizations may be re-weighted under cosmologically informed priors. We list the derivatives of Equation (12) in Appendix A that are required to build the joint covariance for the lensing shear and convergence. We will denote the covariance constructed from second derivatives of Equation (12) as $\mathrm{S}_{\mathrm{GP}}$.

We now return to the pedagogical derivation of the likelihood function for galaxy ellipticity estimators adding the GP distribution for the shear. Because both the marginal likelihood in Equation (8) and the shear prior are Gaussian distributions in the shear, we can specify the shear (and convergence) joint posterior for all galaxy and grid locations as a multivariate Gaussian distribution (otherwise known as the Wiener filter),

$$
\begin{aligned}
\operatorname{Pr}\left(\Upsilon\left(\mathbf{x}, \mathbf{x}^{\prime}\right) \mid \mathbf{d}, \alpha, a_{\mathrm{GP}}\right)=\mathcal{N}_{\Upsilon\left(\mathbf{x}, \mathbf{x}^{\prime}\right)}\left(\boldsymbol{\mu}_{\Upsilon}, \mathrm{S}_{\Upsilon}\right) \\
\\
\times \mathcal{N}_{\hat{\mathrm{e}}}\left(0, \mathrm{~N}+\mathrm{S}_{\mathrm{GP}}\right),
\end{aligned}
$$

where,

$$
\begin{aligned}
& \mathrm{S}_{\Upsilon} \equiv \mathrm{S}_{\mathrm{GP}}\left(\mathrm{S}_{\mathrm{GP}}+\mathrm{N}\right)^{-1} \mathrm{~N} \\
& \boldsymbol{\mu}_{\Upsilon} \equiv \mathrm{S}_{\mathrm{GP}}\left(\mathrm{S}_{\mathrm{GP}}+\mathrm{N}\right)^{-1} \hat{\mathrm{e}} .
\end{aligned}
$$

Calculation of $\boldsymbol{\mu}_{\Upsilon}$ in Equation (16) yields a posterior mean estimate of the lensing shear and convergence at every galaxy location. However, this estimator requires inversion of an $2 n_{\text {gal }} \times 2 n_{\text {gal }}$ covariance matrix, which can be computationally expensive.

Other works have attempted to reduce the dimensionality of the covariances in Equation (14) by interpolating and averaging the measured galaxy ellipticities onto a grid of coarser resolution than that sampled by the galaxy angular distribution (e.g., Alsing et al. 2015). However, such interpolations not only restrict the measured dynamic range, but also can be expected to introduce artefacts in the inferred shear field on the grid based on the shape of the smoothing kernel, to propagate shape measurement systematics to a broad range of angular scales, and to ignore error propagation from individual galaxy shape measurements to the shear inference. Our method defines an explicit interpolation from galaxy to other sky locations, with error propagation included.

We can marginalize the lensing fields at the galaxy locations to obtain the marginal posterior for the lensing 
fields at just the smaller number of grid locations $\mathbf{x}^{\prime}$,

$$
\begin{array}{r}
\operatorname{Pr}\left(\Upsilon\left(\mathbf{x}^{\prime}\right) \mid \mathbf{d}, \alpha, a_{\mathrm{GP}}\right) \propto \int d \Upsilon(\mathbf{x}) \operatorname{Pr}(\mathbf{d} \mid \Upsilon(\mathbf{x}), \alpha) \\
\times \operatorname{Pr}\left(\Upsilon\left(\mathbf{x}^{\prime}\right) \mid \Upsilon(\mathbf{x}), a_{\mathrm{GP}}\right) \operatorname{Pr}\left(\Upsilon(\mathbf{x}) \mid a_{\mathrm{GP}}\right)
\end{array}
$$

When all distributions are Gaussians, Equation (17) reduces to evaluating Equation (14) at only the locations of the grid $\mathbf{x}^{\prime}$ given the input ellipticities ê. Note however that we still require in Equation (16) the evaluation of the shear model at every galaxy location, which is then interpolated by the WF to arbitrary sky locations. Our approach is therefore different from algorithms that require an initial averaging of galaxy ellipticity components over local sky regions. Equation (17) thus specifies the interim distribution of lensing shear and convergence that can be later propagated to cosmological model analyses.

\subsubsection{Optimizing interpolation parameters}

We can further marginalize the lens fields $\Upsilon$ at all locations $\mathbf{x}$ and $\mathbf{x}^{\prime}$ to obtain the marginal likelihood for the GP parameters,

$$
\operatorname{Pr}\left(\mathbf{d} \mid \alpha, a_{\mathrm{GP}}\right)=\mathcal{N}_{\mathbf{d}}\left(0, \mathrm{~S}_{\mathrm{GP}}+\mathrm{N}\right) .
$$

We maximize the density in Equation (18) to determine suitable values of the GP parameters for interpolation of the lens fields for a given ellipticity catalog (see a similar approach in Marshall et al. 2001).

Equation (18) is informative on the GP parameters if the data vector $\mathbf{d}$ includes sufficient numbers of galaxies to beat down the shape noise. In cases with smaller signal to noise ratios we can also add a cosmologically informed prior to help constrain the GP parameters. In this case we can replace the data vector in Equation (18) with a simulation of the data vector derived from a cosmological model. Then, by marginalizing over realizations of the simulated data vector we get a marginal prior for the GP parameters,

$$
\begin{aligned}
\operatorname{Pr}\left(a_{\mathrm{GP}} \mid \theta, \alpha\right) & =\int d \mathbf{d}^{\operatorname{sim}} \operatorname{Pr}\left(a_{\mathrm{GP}} \mid \mathbf{d}^{\operatorname{sim}}, \alpha\right) \operatorname{Pr}\left(\mathbf{d}^{\operatorname{sim}} \mid \theta\right), \\
& =\operatorname{Pr}\left(a_{\mathrm{GP}}\right) \\
& \times \int d \mathbf{d}^{\operatorname{sim}} \frac{\operatorname{Pr}\left(\mathbf{d}^{\operatorname{sim}} \mid a_{\mathrm{GP}}, \alpha\right)}{\operatorname{Pr}\left(\mathbf{d}^{\operatorname{sim}} \mid \alpha\right)} \operatorname{Pr}\left(\mathbf{d}^{\operatorname{sim}} \mid \theta\right) .
\end{aligned}
$$

In Equation (19) we specify a model for drawing simulated data realizations $\mathbf{d}^{\text {sim }}$ given cosmological parameters $\theta$. A standard approach to such a model is to first draw a realization of the $3 \mathrm{D}$ mass density perturbations from a Gaussian distribution with a cosmologically determined initial power spectrum and then to evolve the initial mass density perturbations under a numerical (e.g., $N$-body) simulation of gravitational evolution.

Modeling only linear density perturbation evolution, the late-time model for the projected lensing mass density, and hence the shear, is also a Gaussian distribution,

$$
\operatorname{Pr}\left(\mathbf{d}^{\operatorname{sim}} \mid \theta\right)=\mathcal{N}_{\mathbf{d}^{\operatorname{sim}}}(0, \Sigma(\theta))
$$

for some cosmological covariance $\Sigma(\theta)$. To evaluate
Equation (19) we also must determine the evidence

$$
\operatorname{Pr}\left(\mathbf{d}^{\mathrm{sim}} \mid \alpha, I\right) \equiv \int d a_{\mathrm{GP}} \operatorname{Pr}\left(\mathbf{d}^{\mathrm{sim}} \mid \alpha, a_{\mathrm{GP}}\right) \operatorname{Pr}\left(a_{\mathrm{GP}} \mid I\right),
$$

where $I$ denotes prior information on the GP parameters. Even under a linear density perturbation approximation the evidence cannot be calculated analytically.

For illustration of the GP parameter prior informed by cosmology, we use Equation (20) to evaluate Equation (19) via Monte Carlo integration. We draw $N$ samples of $\mathbf{d}_{i}^{\text {sim }}$ from $\operatorname{Pr}\left(\mathbf{d}^{\operatorname{sim}} \mid \theta\right)$ and evaluate $\operatorname{Pr}\left(\mathbf{d}_{i}^{\text {sim }} \mid a_{\mathrm{GP}}, \alpha\right)$ for each $i=1, \ldots, N$. We approximate the evidence in Equation (21) via 2D numerical integration and then calculate

$$
\operatorname{Pr}\left(a_{\mathrm{GP}} \mid \theta, \alpha\right) \approx \operatorname{Pr}\left(a_{\mathrm{GP}}\right) \frac{1}{N} \sum_{i=1}^{N} \frac{\operatorname{Pr}\left(\mathbf{d}_{i}^{\operatorname{sim}} \mid a_{\mathrm{GP}}, \alpha\right)}{\operatorname{Pr}\left(\mathbf{d}_{i}^{\text {sim }} \mid \alpha, I\right)} .
$$

We show the resulting posterior constraints on the GP parameters in Figure 2. The cosmological simulation re-

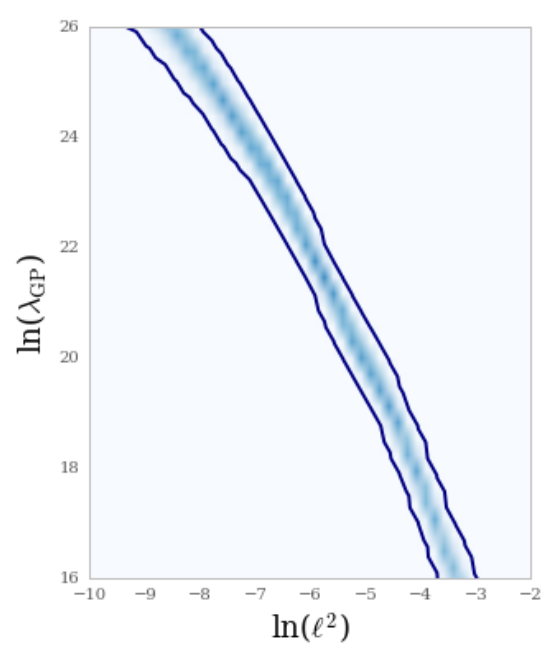

FIG. 2.- Posterior constraints on the GP parameters after marginalizing realizations of simulated lens fields under a cosmological model. The correlations and variance in the simulated data serve to communicate the cosmological covariance structure into constraints on the GP parameters. The tight degeneracy shows the tradeoff between low correlations and large GP precision (equivalent to white noise as the GP precision becomes large) and larger correlations and smaller precision as the simulated maps are fit with smoother models. The lines show the 3- $\sigma$ confidence interval.

alizations impose a tight constraint in a linear combination of the logarithm of the two GP parameters. The constraints in Figure 2 indicate the cosmological lens field simulations can be fit either with a large GP precision and small correlation length (i.e., essentially as white noise), or with a smaller precision and larger correlation lengths (i.e., as a smooth correlated map). We thus obtain tight constraints on the GP parameters if we can include some prior knowledge of the correlation length scale via $\operatorname{Pr}\left(a_{\mathrm{GP}}\right)$.

See Appendix B for a description of our linear theory cosmological model for the lensing convergence.

\subsection{Cosmological parameter inference}


Under a standard cosmological model the lensing potential $\psi$ is related to the $3 \mathrm{D}$ cosmological late-time gravitational potential $\Psi^{\mathrm{LT}}$ by a projection along the line of sight weighted by the lensing efficiency $K\left(a ; A_{s}\right)$,

$$
\bar{\psi}_{s}(\mathbf{x}) \equiv W(\mathbf{x}) \int d z \Psi^{\mathrm{LT}}(\mathbf{x}, z) K\left(z ; Z_{s}\right),
$$

where $W(\mathbf{x})$ is the survey window function, $z$ is the cosmological redshift, and $Z_{s}$ defines the redshift distribution of source galaxies for a galaxy sample $s$. The late-time gravitational potential is in turn related to the potential of the cosmological initial conditions $\Psi^{\mathrm{IC}}$ via a deterministic function $G$ defining gravitational evolution (Jasche \& Wandelt 2013),

$$
\Psi^{\mathrm{LT}}(\mathbf{x}, z)=G\left(\Psi^{\mathrm{IC}}, \theta, z\right) .
$$

We assume the initial conditions $\Psi^{\mathrm{IC}}$ are Gaussian distributed with mean zero and covariance $\Sigma^{\mathrm{IC}}(\theta)$, which is entirely determined by the potential power spectrum from inflation.

A common method to simulate $\Psi^{\mathrm{LT}}$ according to Equation (24) is to run a cosmological $N$-body simulation with initial conditions $\Psi^{\mathrm{IC}}$ drawn from a Gaussian distribution with a specified power spectrum. The numerical $N$ body solver allows evaluation of the function $G$, which does not have a known analytic form beyond low-order perturbation theory. The standard approach to evaluate Equation (23) for the lens potential in a numerical simulation is to trace light rays through the observer's light cone given the simulated $\Psi^{\mathrm{LT}}(\mathbf{x}, a)$. The bundle of light rays is evaluated at a discrete set of sky locations to predict the lensing shear and convergence. The predicted lens fields must then be interpolated over the sky to galaxy locations to complete the numerical cosmological model prediction.

We have already defined a probabilistic interpolation of lens fields over the sky via the GP. We therefore define the conditional distribution of the lens potential at galaxy locations $\mathbf{x}$ given the simulations evaluated at positions $\mathbf{x}^{\prime}$ via the model of Equation (24) and Equation (23) followed by GP interpolation,

$$
\operatorname{Pr}\left(\psi(\mathbf{x}) \mid \bar{\psi}\left(\mathbf{x}^{\prime}, \theta\right), a_{\mathrm{GP}}\right)=\mathcal{N}_{\psi}\left(\mu_{\psi}, \Sigma_{\psi}\right),
$$

where,

$$
\begin{aligned}
\mu_{\psi} & \equiv \mathrm{S}\left(\mathbf{x}, \mathbf{x}^{\prime}\right) \mathrm{S}^{-1}\left(\mathbf{x}^{\prime}, \mathbf{x}^{\prime}\right) \bar{\psi}_{s}\left(\mathbf{x}^{\prime} ; \Psi^{\mathrm{IC}}, \theta, A_{s}, W\right) \\
\Sigma_{\psi} & \equiv \mathrm{S}(\mathbf{x}, \mathbf{x})-\mathrm{S}\left(\mathbf{x}, \mathbf{x}^{\prime}\right) \mathrm{S}^{-1}\left(\mathbf{x}^{\prime}, \mathbf{x}^{\prime}\right) \mathrm{S}\left(\mathbf{x}^{\prime}, \mathbf{x}\right),
\end{aligned}
$$

define the mean and covariance for the conditional multivariate Gaussian. With Equation (25) we have thus derived a conditional probability distribution to compare theoretical predictions of the lens potential with interim samples of the potential drawn under the GP prior. Said another way, our comparison of conditional posterior samples of the lens fields with the cosmological models is mediated by the interpolation over the sky using the GP.

By marginalizing the initial conditions realizations and the lens fields realizations we can now derive the posterior distribution for the cosmological parameters,

$$
\begin{aligned}
& \operatorname{Pr}\left(\theta \mid \mathbf{d}, a_{\mathrm{GP}}, \alpha\right) \propto \operatorname{Pr}(\theta) \\
& \times \int d \Upsilon \int d \Upsilon^{\prime} \int d \Psi^{\mathrm{IC}} \delta_{D}\left(\Upsilon^{\prime}-h\left(\Psi^{\mathrm{IC}}\right)\left(\mathbf{x}^{\prime}\right)\right) \\
& \quad \times \frac{\operatorname{Pr}\left(\Psi^{\mathrm{IC}}\left(\mathbf{x}^{\prime}\right) \mid \Sigma^{\mathrm{IC}}(\theta)\right)}{\operatorname{Pr}\left(\Upsilon^{\prime} \mid a_{\mathrm{GP}}\right)} \\
& \quad \times \operatorname{Pr}(\mathbf{d} \mid \Upsilon, \alpha) \operatorname{Pr}\left(\Upsilon \mid \Upsilon^{\prime}, a_{\mathrm{GP}}\right) \operatorname{Pr}\left(\Upsilon^{\prime} \mid a_{\mathrm{GP}}\right),
\end{aligned}
$$

where $h\left(\Psi^{\mathrm{IC}}\right)$ indicates the deterministic gravitational evolution of the initial conditions potential to late times where the lensing is observed.

The final line of Equation (28) is the interim sampling distribution we defined in the previous section. We can thus perform the integrals in Equation (28) via Monte Carlo with the same interim lens field samples that are generated in the map making algorithm.

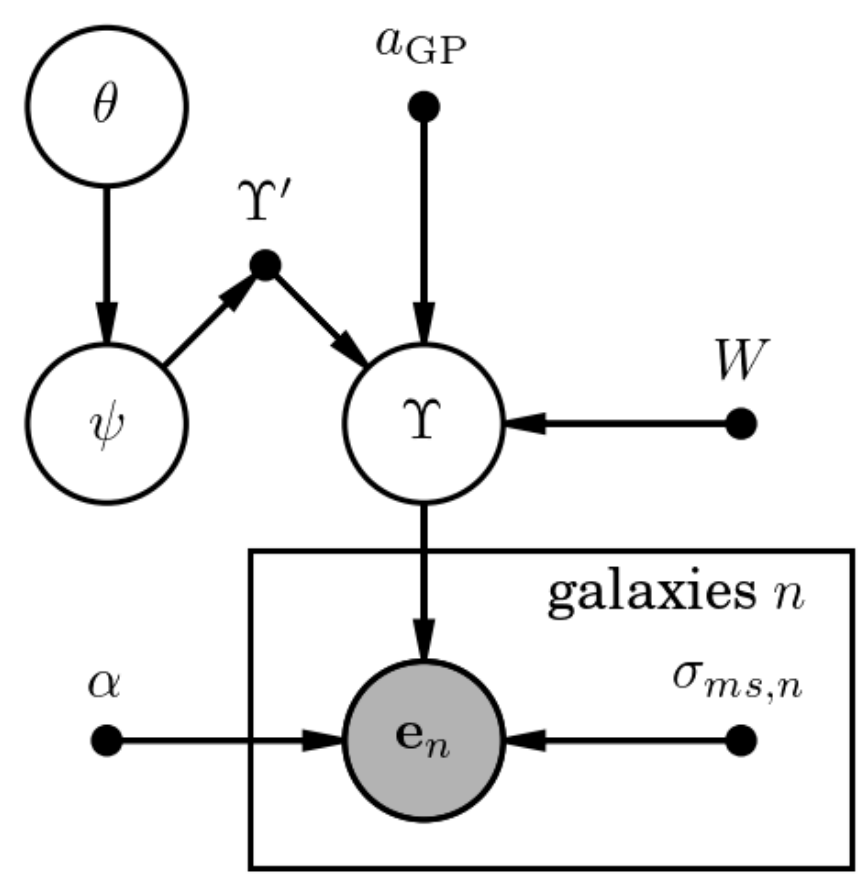

FIG. 3.- In our approximate cosmological parameter inference pipeline we assume the lens fields at the galaxy locations $\Upsilon$ are entirely determined by GP interpolation from the lens fields at a regular set of sky locations $\Upsilon^{\prime}$ at which we compute theory predictions. The lens fields on the regular set are informed by the cosmological model with parameters $\theta$.

We propose two sampling algorithms depending on whether or not we can directly evaluate the probability density for the lens fields under the cosmological model.

Sampling algorithm 1: This is an algorithm to use when it is possible to analytically evaluate the cosmological probability density function. With $K$ samples of $\Upsilon\left(\mathbf{x}^{\prime}\right)$ from the interim sampling distribution, we use importance sampling to approximate the marginalizations in Equation (28),

$$
\operatorname{Pr}\left(\theta \mid \mathbf{d}, a_{\mathrm{GP}}, \alpha\right) \approx \operatorname{Pr}(\theta) \frac{1}{K} \sum_{k=1}^{K} \frac{\operatorname{Pr}\left(\Upsilon_{k}\left(\mathbf{x}^{\prime}\right) \mid \theta\right)}{\operatorname{Pr}\left(\Upsilon_{k}\left(\mathbf{x}^{\prime}\right) \mid a_{\mathrm{GP}}\right)},
$$


where from Equation (28),

$$
\begin{aligned}
\operatorname{Pr}\left(\Upsilon\left(\mathbf{x}^{\prime}\right) \mid \theta\right) \equiv \int d \Psi^{\mathrm{IC}} & \operatorname{Pr}\left(\Psi^{\mathrm{IC}}\left(\mathbf{x}^{\prime}\right) \mid \Sigma^{\mathrm{IC}}(\theta)\right) \\
& \times \delta_{D}\left(\Upsilon^{\prime}-h\left(\Psi^{\mathrm{IC}}\left(\mathbf{x}^{\prime}\right)\right)\right) .
\end{aligned}
$$

Sampling algorithm 2: This is an algorithm to use when cosmological modeling of the lens fields is only possible via forward simulation. For most cosmological models of interest, we can only predict the model for the lens fields via forward simulation of the cosmological mass density perturbation evolution. In this case, we have no direct mechanism to evaluate the density $\operatorname{Pr}\left(\Upsilon\left(\mathbf{x}^{\prime}\right) \mid \theta\right)$. Instead, we follow the steps,

1. Draw $\Psi^{\mathrm{IC}}\left(\mathbf{x}^{\prime}\right)$ from $\operatorname{Pr}\left(\Psi^{\mathrm{IC}}\left(\mathbf{x}^{\prime}\right) \mid \Sigma^{\mathrm{IC}}(\theta)\right)$.

2. Compute the predicted lens fields given the drawn initial conditions $h\left(\Psi^{\mathrm{IC}}\right)$ (e.g., via $N$ body simulation and ray-tracing prediction of the lensing shear and convergence).

3. Select $K$ samples of $\Upsilon(\mathbf{x})$ from the set of interim samples under the GP prior.

4. Evaluate the density, $\operatorname{Pr}\left(\Upsilon \mid \Upsilon^{\prime}, a_{\mathrm{GP}}\right)$, as in Equation (25) for each sample $K$ and compute,

$\operatorname{Pr}\left(\theta \mid \mathbf{d}, a_{\mathrm{GP}}, \alpha\right) \approx \operatorname{Pr}(\theta) \frac{1}{K} \sum_{k=1}^{K} \operatorname{Pr}\left(\Upsilon_{k}(\mathbf{x}) \mid \Upsilon\left(\mathbf{x}^{\prime}, \theta\right), a_{\mathrm{GP}}\right)$.

Note that $\Upsilon^{\prime}$ here is that from the cosmological forward model, not that from the interim sampling.

For Sampling Algorithm 1, if we assume linear cosmological perturbations we can approximate,

$$
\operatorname{Pr}\left(\Upsilon\left(\mathbf{x}^{\prime}\right) \mid \theta\right) \approx \mathcal{N}_{\Upsilon\left(\mathbf{x}^{\prime}\right)}\left(\mathbf{0}, \Sigma\left(P_{\Upsilon}(\theta)\right)\right) .
$$

Under this approximation we can combine the terms from Equation (28),

$\operatorname{Pr}\left(\Upsilon \mid \Upsilon^{\prime}, a_{\mathrm{GP}}\right) \operatorname{Pr}\left(\Upsilon^{\prime} \mid \theta\right)=\mathcal{N}_{\Upsilon\left(\mathbf{x}, \mathbf{x}^{\prime}\right)}\left(0, \mathrm{M}\left(\mathbf{x}, \mathbf{x}^{\prime} ; a_{\mathrm{GP}}, \theta\right)\right)$,

where $\mathrm{M}\left(\mathbf{x}, \mathbf{x}^{\prime} ; a_{\mathrm{GP}}, \theta\right)$ is defined in Equation $\mathrm{C} 7$.

If we further assume, as above, that the likelihood function is a Gaussian distribution in the galaxy ellipticities with a linear weak shear applied, then Equation (28) becomes,

$$
\operatorname{Pr}\left(\theta \mid \mathbf{d}, a_{\mathrm{GP}}, \alpha\right) \propto \operatorname{Pr}(\theta) \mathcal{N}_{\mathbf{d}}\left(0, \mathrm{M}\left(a_{\mathrm{GP}}, \theta\right)+\mathrm{N}\right),
$$

with the signal covariance as defined in Equation C7.

To summarize, the approximations required for Equation (34) are,

1. a likelihood function that is Gaussian in the galaxy ellipticities,

2. galaxy ellipticity measurements that are unbiased estimators of the reduced shear,

3. weak shear $(\kappa \ll 1)$,

4. linear cosmological perturbations.
We can drop assumptions 1, 2, and 3 and still use Sampling Algorithm 1. But to avoid assumption 4 we must resort to Sampling Algorithm 2.

\section{RESULTS}

We evaluate the mean and covariance of the marginal posterior for the lensing shear and convergence from Equation (14) for a simulated data set, where we can compare with a known truth (subsection 3.1). We also analyze the ellipticity catalog (Jee et al. 2013) in the Deep Lens Survey (DLS) ${ }^{7}$ (Wittman et al. 2002) in the vicinity of a massive galaxy cluster where the amplitudes of the lensing fields are large (subsection 3.2).

\subsection{Simulation study}

To validate that Equation (16) can recover the correct shear and convergence fields, we create simulated galaxy ellipticity catalogs with artificially small shape noise so we can measure the lensing shear and convergence to a tuneable precision.

Our procedure for simulating galaxy ellipticity catalogs is,

1. Calculate a lensing convergence angular power spectrum using the cosmology theory code $\mathrm{CHOMP}^{8}$ (Morrison \& Schneider 2013). We assume a standard $\Lambda$ CDM cosmology with $\Omega_{m}=0.3$, $\sigma_{8}=0.8$ and a source redshift distribution with a narrow peak at $z=1$.

2. Simulate Gaussian-distributed lensing shear and convergence maps on a grid using the code GAL$\mathrm{SIM}^{9}$ (Rowe et al. 2015).

3. Place one galaxy in each grid cell of the simulated lensing shear maps. These galaxies are simply sources of illumination for measuring the lensing fields, not cosmologically clustered galaxies.

4. For each galaxy, draw intrinsic ellipticity components from a 2D Gaussian distribution with mean 0 and a specified variance, $\sigma_{e}^{2}$.

5. Calculate lensed ellipticities for each galaxy by adding the lensing shear to the intrinsic ellipticities, assuming a weak shear approximation.

6. Save the galaxy angular sky locations and ellipticity components to a catalog file.

Given a simulated ellipticity catalog, we find the GP parameters $\lambda_{\mathrm{GP}}, \ell_{\mathrm{GP}}^{2}$ that maximize Equation (18). We then evaluate Equation (16) and Equation (15) using the optimized GP parameters to obtain the marginal posterior distribution of the lensing fields $\kappa, \gamma$ at all galaxy locations as well as on a regular grid of locations. We expect that in practical applications to large data sets the shear will only need to be evaluated at the galaxy locations and the converngence (or lens potential) will only need to be evaluated at a smaller number of sky grid locations, thus reducing the overall dimensionality of the linear system to be solved.

\footnotetext{
7 http://dls.physics.ucdavis.edu

8 https://github.com/karenyyng/chomp

9 https://github.com/GalSim-developers/GalSim
} 
We show an example of the output of this procedure in Figure 4 compared to the input shear fields used to generate the mock ellipticity catalog. In the left column in Figure 4 we show the posterior mean fields from Equation (16). In the adjacent panel we show the input shear fields. We show the 'true' convergence that we calculated at the same time as the input shear, but we do not use the convergence at any point in our calculation. The "estimated convergence" comes from interpolating the measured galaxy ellipticities with the GP kernel. The right panel in Figure 4 shows the signal-to-noise ratio (SNR) of the lens field maps, defined as,

$$
\mathrm{SNR} \equiv \Upsilon / \sqrt{\operatorname{diag}\left(\mathrm{S}_{\Upsilon}\right)}
$$

with $\mathrm{S}_{\Upsilon}$ defined in Equation (15). In the limit that the variance of the lens fields dominates the intrinsic shape and ellipticity measurement variances, Equation (15) reduces to $\mathrm{N}$. Then Equation (35) becomes $\mathrm{SNR} \rightarrow \Upsilon / \sqrt{\sigma_{e}^{2}+\sigma_{\mathrm{ms}}^{2}}$, which is similar to SNR definitions in other weak lensing mass mapping analyses (van Waerbeke 2000; Shan et al. 2014).

The simulation in Figure 4 has an artificially low intrinsic ellipticity r.m.s. of $\sigma_{e}=0.0026$ compared to $\sigma_{e}=0.26$ in the Deep Lens Survey that we analyze in subsection 3.2. We choose a small shape noise r.m.s. to allow us to validate our convergence inference with a small number of only 1600 galaxies. We place the simulated galaxies on a $40 \times 40$ grid, so that we do not have to interpolate the simulated shear fields to build the mock ellipticity catalog.

Because the Gaussian model for the shape noise r.m.s. scales with the number of galaxies $n_{\text {gal }}$ as $n_{\text {gal }}^{-1 / 2}$, the shape noise r.m.s. in our simulation is equivalent to a DLS-like galaxy sample with 16 million galaxies. This is about 64 times the number of galaxies we have in a single four square degree field of the DLS. We show the effect of increasing the shape noise r.m.s. by a factor of $\sqrt{64}$ later in this section.

The reconstructed shear and convergence maps in Figure 4 are a good match to the simulation inputs, but are noticeably smoothed. This is for two reasons; (1) we evaluate the interpolated lens fields on a smaller $24 \times 24$ grid than the $40 \times 40$ grid on which the inputs are evaluated, (2) we use a value of $\ell_{\mathrm{GP}}^{2}=0.0123$ that is larger than the Nyquist scale in the maps, even for the $24 \times 24$ interpolated grid because of the way the GP parameters are optimized. We will discuss the GP parameter optimization below.

In Figure 5 we show the E and B mode power spectrum estimators obtained from the posterior mean shear fields shown in Figure 4. We compare the E-mode power spectrum estimator from the mean posterior shear maps with that using the higher-resolution shear maps that were used to generate the mock ellipticity catalog (which we label 'simulation truth'). We also show in Figure 5 the 'theory' power spectrum that we used to generate the 'simulation truth' shear maps. The 'theory' and 'simulation truth' spectra agree on scales below the Nyquist frequency, shown in Figure 5 as the vertical blue line. The mean posterior power spectrum agrees with the 'simulation truth' spectrum on scales below the effective smoothing frequency derived from the value of $\ell_{\mathrm{GP}}^{2}$ and shown by the vertical dot-dashed line in Figure 5.

The B-mode power spectrum estimated from the mean posterior shear maps is shown by the dashed red line in Figure 5. We expect the B-mode power to be consistent with zero because we simulated only E-mode power. The nonzero B-mode power spectrum in Figure 5 is explained by examining Figure 6, which shows the $\mathrm{E}$ and $\mathrm{B}$ mode mean posterior maps from which the $\mathrm{E}$ and $\mathrm{B}$ mode mean posterior power spectra were derived. The B-mode map in Figure 6 is near zero throughout the field except near the boundaries. These edge effects in the B-mode map can be explained by mathematical ambiguities in the definition of $\mathrm{E}$ and $\mathrm{B}$ mode separation in a finite field (Bunn et al. 2003). The small value of the B-mode map in Figure 6 away from the field boundaries indicates our GP interpolation method does not create spurious B-modes.

To illustrate the GP parameter optimization procedure we show in Figure 7 the mean posterior convergence, the associated SNR maps, and the marginal likelihood surface for the GP parameters (left to right columns) for increasing intrinsic ellipticity variance $\sigma_{e}^{2}$ (top to bottom rows). The top row of panels in Figure 7 show a repeat of the $\sigma_{e}^{2}$ and $a_{\mathrm{GP}}$ values used in Figure 4 . For the very small shape noise in this case, there is a narrow peak in the log-likelihood surface for the two GP kernel parameters. We thus select the maximum likelihood (ML) values for $a_{\mathrm{GP}}$ and obtain the convergence and SNR maps that closely resemble the 'true' convergence as shown in Figure 4. However, the ML value of $\ell_{\mathrm{GP}}^{2}$ is somewhat larger than the Nyquist frequency of the grid to which we interpolate as shown in Figure 5 (compare dot-dashed to red solid vertical lines). Because the noise is sub-dominant in this example, we would expect a value of $\ell_{\mathrm{GP}}^{2}$ matching the grid Nyquist frequency to yield a more accurate convergence map reconstruction. We are likely to obtain more accurate results, therefore, if we impose a prior on $a_{\mathrm{GP}}$ that encodes this expectation.

As the shape noise increases (for the same input signal and measurement uncertainties), the peak in the marginal log-likelihood surface for $a_{\mathrm{GP}}$ becomes broader and eventually disappears as shown by the rows of panels from top to bottom in Figure 7. The ML value for $\ell_{\mathrm{GP}}^{2}$, while less well defined, continues to yield maps that are more smoothed as $\sigma_{e}^{2}$ increases. This helps to preserve large amplitudes of the peaks in the SNR maps (see the color bar scales in the SNR maps of Figure 7), but in compensation erases structures at all but the lowest spatial frequencies in the maps. This procedure, with flat priors in the log of $a_{\mathrm{GP}}$, appears useful for visualizing the posterior convergence maps, but is undesirable for subsequent cosmological analyses. We see again that we would prefer a prior favoring smaller $\ell_{\mathrm{GP}}^{2}$ even as the shape noise becomes large.

We assert such a prior in Figure 8, by imposing Gaussian priors separately in $\ln \left(\lambda_{\mathrm{GP}}\right)$ and $\ln \left(\ell_{\mathrm{GP}}^{2}\right)$ with parameters given in Table 2. Our Gaussian prior is informed by the cosmological simulation study shown in Figure 2 combined with our prior that the GP correlation length be large enough so that white noise does not dominate the fits to the lens fields. For each value of $\sigma_{e}$ in Figure 8 we see that the signal-to-noise ratio (SNR) is comparable to that in Figure 7 but the convergence 

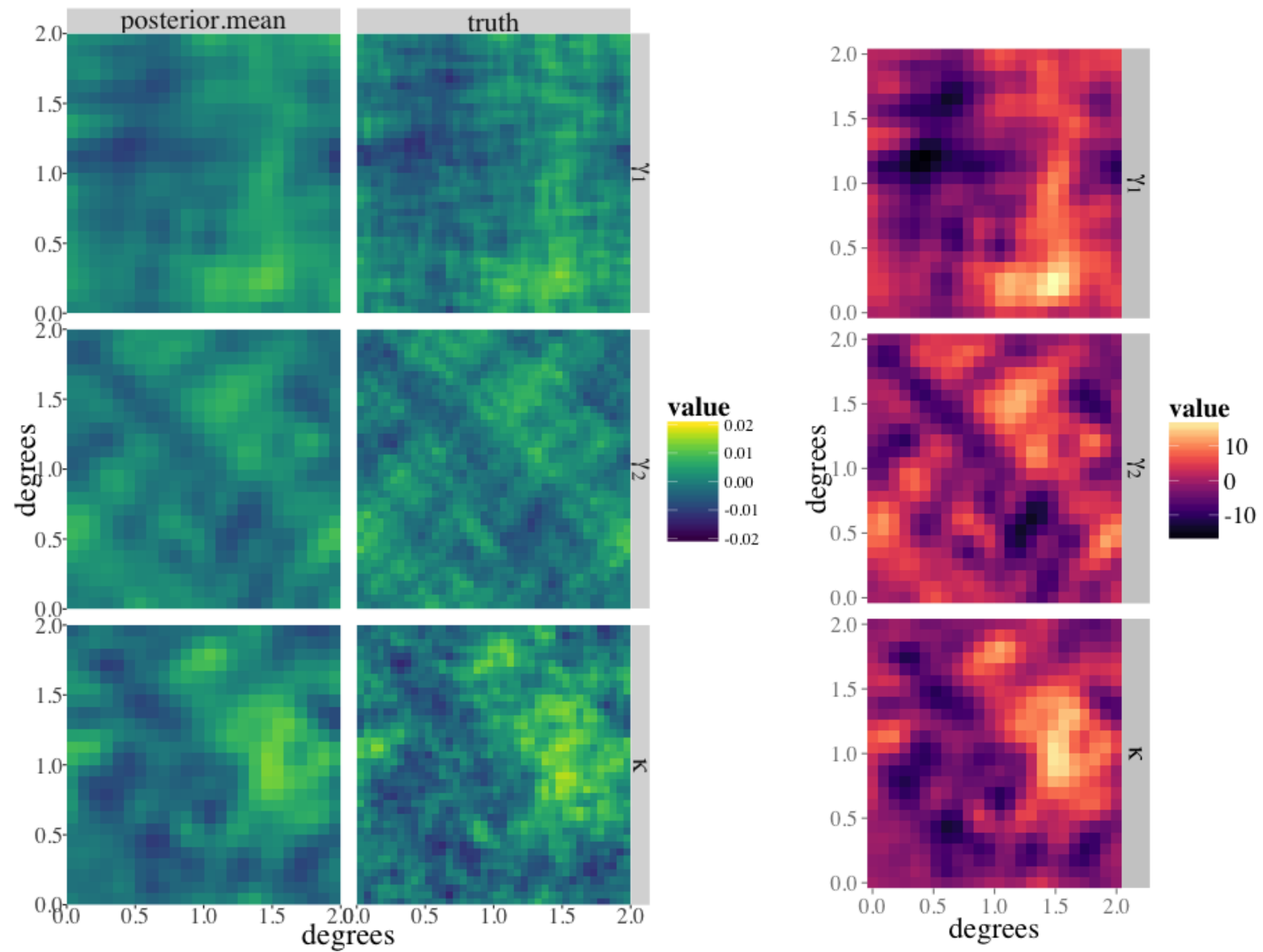

FIG. 4.- Left: Comparison of the convergence and shear maps in our simulation study between that used to generate our mock galaxy ellipticity catalog (right column) and the output of our GP interpolation (left column). The rows show the maps for the two shear components $\gamma_{1,2}$ and the convergence $\kappa$. These maps cover a $2 \times 2$ square degree field. The simulated intrinsic ellipticity r.m.s. is set to an artificially small value of $\sigma_{e}=0.0026$, which is 100 times smaller than that observed for the complete Deep Lens Survey catalog. Right: signal-to-noise ratio (SNR) maps for the same simulations. We calculate SNR as the ratio of the map to the square root of the diagonal of the covariance in Equation (15). These simulations use 1600 galaxies and grid sizes for the GP interpolated shear and convergence with 24 grid cells per dimension.

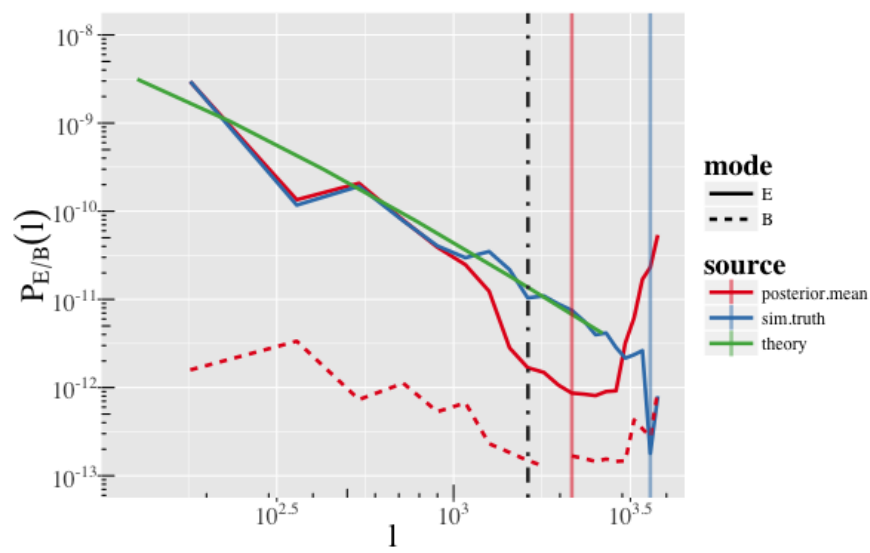

FIG. 5.- Comparison of power spectrum estimators in our simulation study to the 'truth' input spectrum. The vertical solid lines show the Nyquist frequencies of the grids. The vertical dot-dashed line shows the multipole corresponding to the length scale $\sqrt{\ell_{\text {GP }}^{2}}$ set by the GP kernel parameter $\ell_{\mathrm{GP}}^{2}=0.0123$ for field coordinates are normalized to the unit square.

maps include higher spatial frequency structures.

\subsubsection{Cosmological parameter constraints}

TABLE 2

PARAMETERs For the GaUSSiAn PRIOR ON THE GP PARAMETERS FOR OUR SIMULATION STUDY.

\begin{tabular}{lcc}
\hline \hline Parameter & mean & std. dev. \\
\hline $\ln \left(\lambda_{\mathrm{GP}}\right)$ & 18 & 0.43 \\
$\ln \left(\ell_{\mathrm{GP}}^{2}\right)$ & -4.0 & 0.1 \\
\hline \hline
\end{tabular}

Although we assert a non-cosmological GP prior on the lens fields to infer regular convergence and shear maps, we now demonstrate how we can recover cosmological information in a manner similar to common algorithms in the literature. That is, we compute an angular power spectrum estimator for the lensing convergence from the lens field posterior distribution.

The posterior distribution for the lens fields given the GP parameters is a multivariate Gaussian distribution characterized by a mean field and covariance as given in Equation (16) and Equation (15). The posterior mean is thus a convenient and useful summary statistic (as we have shown above). Also, because an isotropic Gaussian random field is fully described by the angular power spectrum, it is common in the literature to reduce cosmological large-scale structure statistics to two-point function estimators for cosmological parameter estimation. 


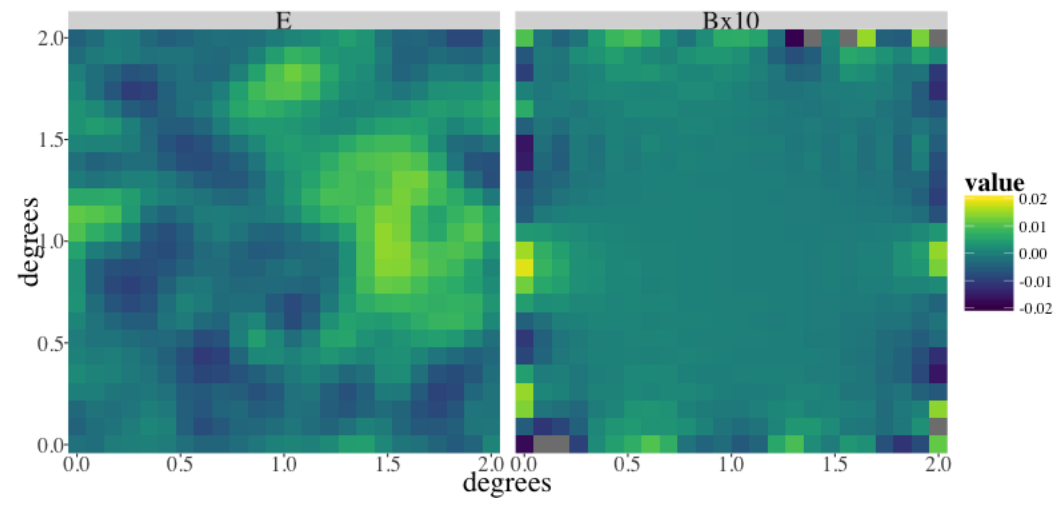

FiG. 6. - E and B mode maps derived from the posterior mean shear maps shown in the left column of Figure 4. The E-mode map closely matches the interpolated convergence map in Figure 4 as expected. The B-mode map is near zero throughout the center of the field, but shows non-zero values around the field edge because of mathematical ambiguities in the E/B mode separation at the field boundaries (Bunn et al. 2003). Note the value of the plotted B-mode map has been multiplied by 10 for easier visualization.

We showed the angle-averaged (E-mode) convergence power spectrum estimator for our low-noise Gaussian simulated maps in Figure 5. We use this power spectrum estimator as a summary statistic derived from the observed ellipticity catalog. We further assert a multivariate Gaussian likelihood function for the power spectrum estimator with covariance (e.g., Cooray \& Hu 2001),

$$
\operatorname{Cov}\left(P_{\kappa}(\ell)\right)=\frac{2}{N_{\ell}} P_{\kappa}^{2}(\ell),
$$

where $N_{\ell}$ is the number of modes contributing to the band power estimator for a multipole bin centered at $\ell$.

In Figure 9 we show $68 \%$ and $95 \%$ contours of the 2D posterior distribution for the cosmological mass density $\Omega_{m}$ and density fluctuations r.m.s. $\sigma_{8}$ given the angular power spectrum likelihood just described and flat priors. The values used to generate the mock data are $\Omega_{m}=$ $0.3, \sigma_{8}=0.8$. We limit the multipole range of the power spectrum in the likelihood to $100<\ell<1300$, where the upper bound is set by the effective smoothing length imposed by the asserted GP correlation parameter $\ell_{\mathrm{GP}}^{2}$.

We see from Figure 9 that the GP interim prior used to derive the convergence map from the galaxy ellipticity catalog has not biased the cosmological parameter constraints (within the uncertainties) obtained from a reduced summary statistic of the lens field posterior. We defer to later work more complete demonstrations of the cosmological parameter inference algorithms in subsection 2.2 based on marginalizing the lens field realizations.

\subsection{Abell cluster in the Deep Lens Survey}

We apply our lens field inference algorithm to the galaxy ellipticity catalog derived from the $\operatorname{DLS}^{10}$ (Wittman et al. 2002). The DLS is a 20 square degree optical imaging survey optimized for cosmic shear measurements. We analyze a $\sim 1$ square degree field centered on the Abell cluster 781, which was previously analyzed using DLS lensing shear measurements in Wittman et al. (2006); Kubo et al. (2009); Wittman et al. (2014). Abell 781 consists of four massive galaxy clusters, which is a useful case study for our algorithm because it provides a large lensing signal with a modest number of galaxies and because the distribution of

10 http://dls.physics.ucdavis.edu mass density perturbations is decidedly not Gaussian distributed.

Jee et al. (2013) presented galaxy ellipticity measurements with the DLS $R$-band imaging calibrated to produce shear estimates with biases well below the statistical uncertainties for two-point cosmic shear correlation function estimators. The DLS shear pipeline in Jee et al. (2013) includes correlated PSF size and ellipticity corrections in each DLS exposure, calibration of additive and multiplicative shear biases with image simulations, and a set of null tests validating the PSF shear calibration corrections. The shear estimation pipeline used in Jee et al. (2013), sFIT, was further validated as the winning algorithm in the blinded community shear measurement challenge GREAT3 (Mandelbaum et al. 2015). The DLS was performed with four optical pass-bands $(B V R z)$ that allow photometric redshift (photo- $z$ ) estimates for all lensing source galaxies (Schmidt \& Thorman 2013). Jee et al. (2016) extended the DLS shear analysis to a tomographic cosmic shear measurement using the photo- $z$ estimates.

The DLS galaxy ellipticity catalog produced for Jee et al. (2013) includes a catalog-level selection based on measured galaxy magnitudes, sizes, ellipticity measurement error, photo- $z$ estimates, and proximity to masks as listed in Jee et al. (2013) Table 2. We perform a further set of selections on this catalog as listed in Table 3 . We

TABLE 3

Selection CRiteria APplied to the DLS Galaxy CATAlog.

\begin{tabular}{ccc}
\hline \hline Parameter & $\min$ & $\max$ \\
\hline$z_{b}$ & 0.45 & - \\
$d e$ & - & 0.1 \\
$\sqrt{a^{2}+b^{2}}$ & $0.8^{\prime \prime}$ & - \\
$R$ & 22 & 23 \\
\hline \hline
\end{tabular}

choose the lower bound on (maximum posterior) photo- $z$, $z_{b}$, to select source galaxies that are likely to be at redshifts larger than that of the highest redshift sub-cluster in the field at $z \approx 0.43$ (Wittman et al. 2014). We further select only those galaxies in the ellipticity catalog with ellipticity measurement errors, de, less than 0.1 and sizes greater than 0.8 arcseconds to obtain galaxies likely to have more precisely measured shapes for informing the lensing shear. We exclude galaxies with sizes, as deter- 

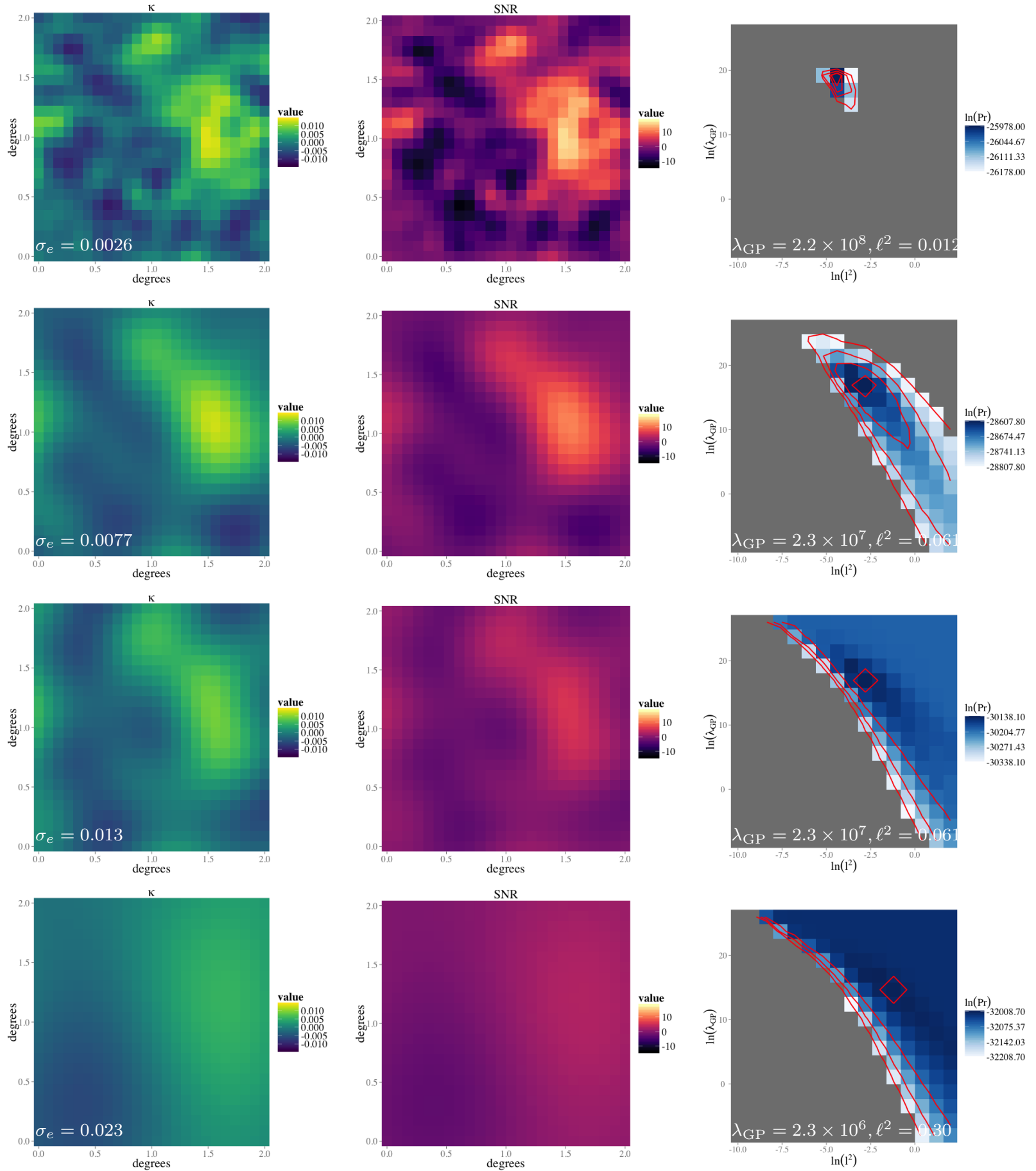

FIG. 7.- Posterior mean convergence maps (left), signal-to-noise ratio (SNR) maps (middle), and marginal likelihood contours (right) for simulated galaxy catalogs with varying intrinsic ellipticity r.m.s. $\sigma_{e}$. From top to bottom, $\sigma_{e}=(0.00258,0.00774,0.0129,0.0232)($ these values are also annotated in the bottom left corner of each convergence map). The top row matches the simulation shown in Figure 4 . Following the right column from top to bottom shows how the maximum-likelihood estimates for the GP parameters shifts to longer correlation lengths and smaller precision parameters with increasing shape noise. That is, as the data becomes more noise dominated, the marginal GP parameter likelihood changes shape to prefer smoothing, or effectively averaging, more galaxies to retain a more significant shear and convergence signal. The ML values for the GP parameters are listed in each panel showing the log-likelihood contours. 

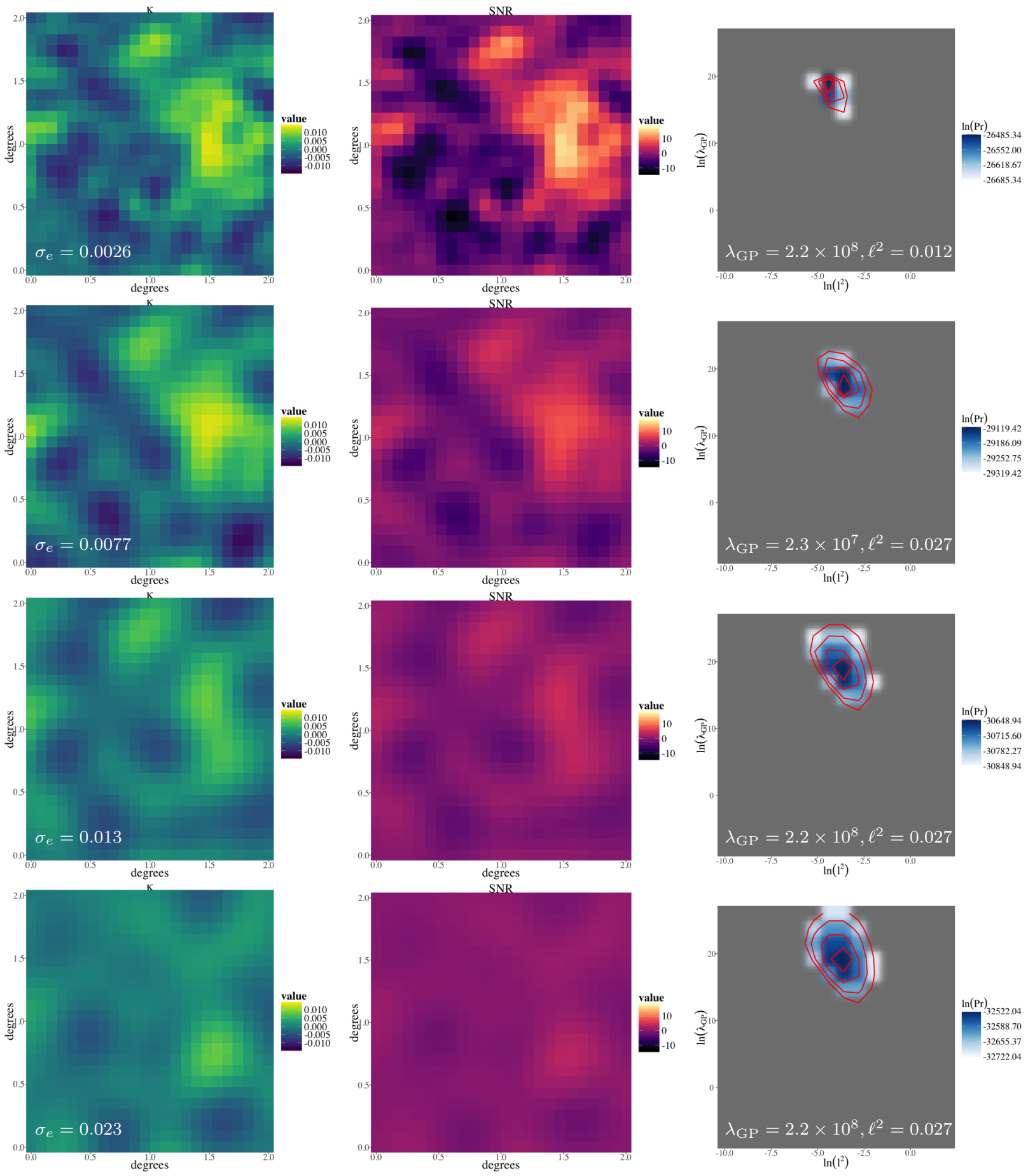

FIG. 8. - Same as Figure 7 except with a prior asserted for the GP parameters $a_{\mathrm{GP}}$ that limits the degree of smoothing in the posterior mean maps. 


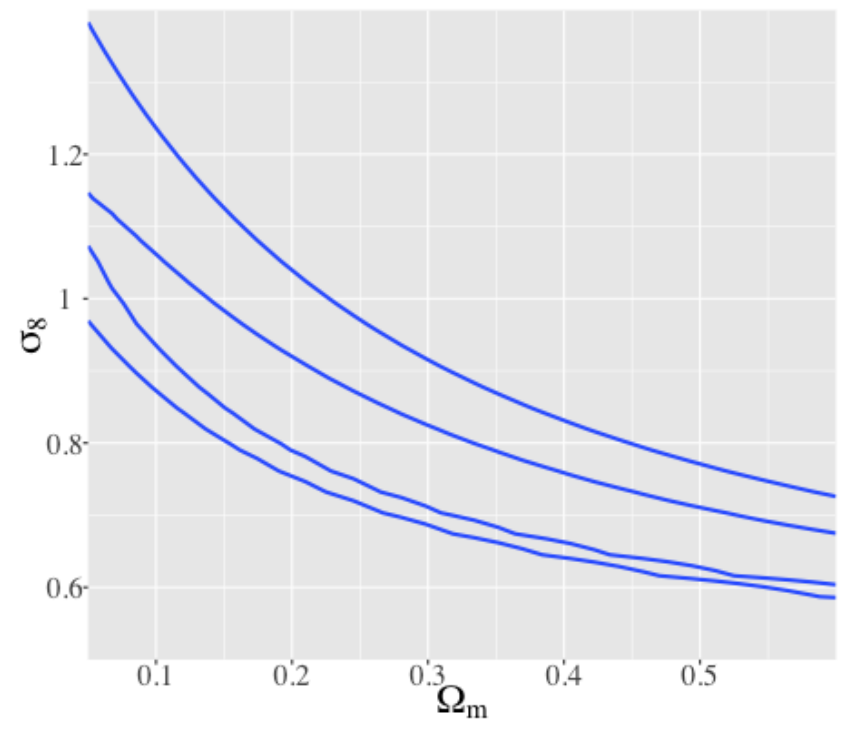

FIG. 9.- Marginal constraints on the cosmological parameters using the E-mode power spectrum estimator derived from the mean posterior convergence field.

mined from the geometric mean of the semi-major and semi-minor axes $a, b$, less than 0.8 arcseconds because the ellipticities tend to be less well measured when the galaxy size is similar to that of the PSF. We select the brighter galaxies based on $R$-band magnitude that are still likely to be faint enough to avoid significant contamination from cluster members. After all the selections listed in Table 3 we measure an ellipticity r.m.s. of $\sigma_{e}=0.21$. This measurement includes the ellipticities with lensing effects included, but because lensing is subdominant to the intrinsic ellipticity dispersion we assert $\sqrt{\alpha}=\sigma_{e}=0.21$ for the posterior inference for A781.

We show our mean posterior inference of the lensing convergence of A781 in Figure 10 using 6000 galaxies randomly selected from the cut sample described in Table 3 . We select only 6000 galaxies to limit the size of the lens field joint covariance that we must invert. The left column of panels shows the convergence while the right column shows the signal-to-noise ratio for the mean posterior. Our calculation is in the top row of panels in Figure 10, which we compare with the aperture mass algorithm of Dawson et al. (2012) in the bottom row of panels. Note we use the same galaxy sample as input to each mass mapping algorithm. The algorithm of Dawson et al. (2012), called 'aperture densomitry', provides a mass estimator that is more localized on the sky. The shear in apertures is averaged with weighting functions that account for both angular selections and the expected line-of-sight lensing kernel with the aide of the photometric redshift information in the DLS catalog. However, to make a more direct comparison with the algorithm in this paper, we recomputed the aperture densomitry weights without using any photometric redshift information for the source galaxies.

Figure 10 shows that we obtain consistent results for the two main A781 sub-clusters using our mean posterior map and the method used in a previous analysis. The white crosses in Figure 10 indicate the locations of all sub-clusters detected in Wittman et al. (2014). We do not detect all the same sub-clusters, which is likely be- cause we use a significantly smaller number of galaxies (6000 versus $\sim 50000$ ) while we test the performance and scaling of our codes. However one sub-cluster denotedin Figure 10 is only detected in the literature in x-ray emission (Sehgal et al. 2008). Wittman et al. (2014) also weight the source galaxies according to the expected lensing kernel and the photo- $z$ estimates. We make no use of photo- $z$ information other than in the sample selection.

TABLE 4

Parameters for the Gaussian PRior on the GP parameters FOR A781.

\begin{tabular}{lcc}
\hline \hline Parameter & mean & std. dev. \\
\hline $\ln \left(\lambda_{\text {GP }}\right)$ & $\ln \left(10^{6}\right)$ & 0.1 \\
$\ln \left(\ell^{2}\right)$ & $\ln \left(10^{-4}\right)$ & 0.5 \\
\hline \hline
\end{tabular}

In Figure 11 we show the marginal log-likelihood for the A781 ellipticities in the plane of the two GP parameters. Unlike in Figure 7 the noise covariance is now significant in defining the contours in Figure 11 such that a large GP precision and small GP correlation length is favored (indicating a sub-dominant signal covariance). We therefore impose a Gaussian prior in the logarithm of the GP parameters with parameters listed in Table 4. We infer maximum posterior values of $\lambda_{\mathrm{GP}}=2.7 \times 10^{6}$ and $\ell^{2}=0.012$, which we use in the convergence inference in Figure 10. However, as shown in Figure 11, the marginal posterior for the GP parameters is only weakly peaked for this data set, and a range of GP parameters would be acceptable for the mass map inference.

\section{CONCLUSIONS}

We have demonstrated a probabilistic model inference of lensing shear and convergence using a Gaussian Process (GP) prior. Our method is an extension of previous Bayesian maximum entropy mass mapping methods that is applicable in both cosmic shear (i.e., 'field') and cluster lensing regimes. We validated our algorithm using simulated Gaussian distributed shear maps with comparisons to the input 'truth' and by reconstructing the mass distribution in a cluster field in the Deep Lens Survey where we compare with the results of a previously published 'aperture densometry' method.

Because our GP kernel for the lensing shear and convergence is derived from a consistent lens potential, we find excellent separation of $\mathrm{E}$ and $\mathrm{B}$ modes in the posterior lens field maps. Recently ? presented an algorithm to project Wiener Filter maps into 'pure' E and B modes, removing ambiguity in the $\mathrm{E} / \mathrm{B}$ decomposition from survey masks. Because we compute Wiener Filter solutions for the lensing convergence from galaxy ellipticities, the method of ? is a simple extension of the algorithm we present in this paper.

We have also described an algorithm for optimization of the GP kernel parameters given a measured galaxy ellipticity catalog or a cosmologically-informed prior based on simulation of lensing fields. An interesting future extension of this work could include both galaxy positions and ellipticities in the reconstruction of the gravitational potential.

Our algorithm is computationally challenging in the solution of linear systems of dimension equal to the 

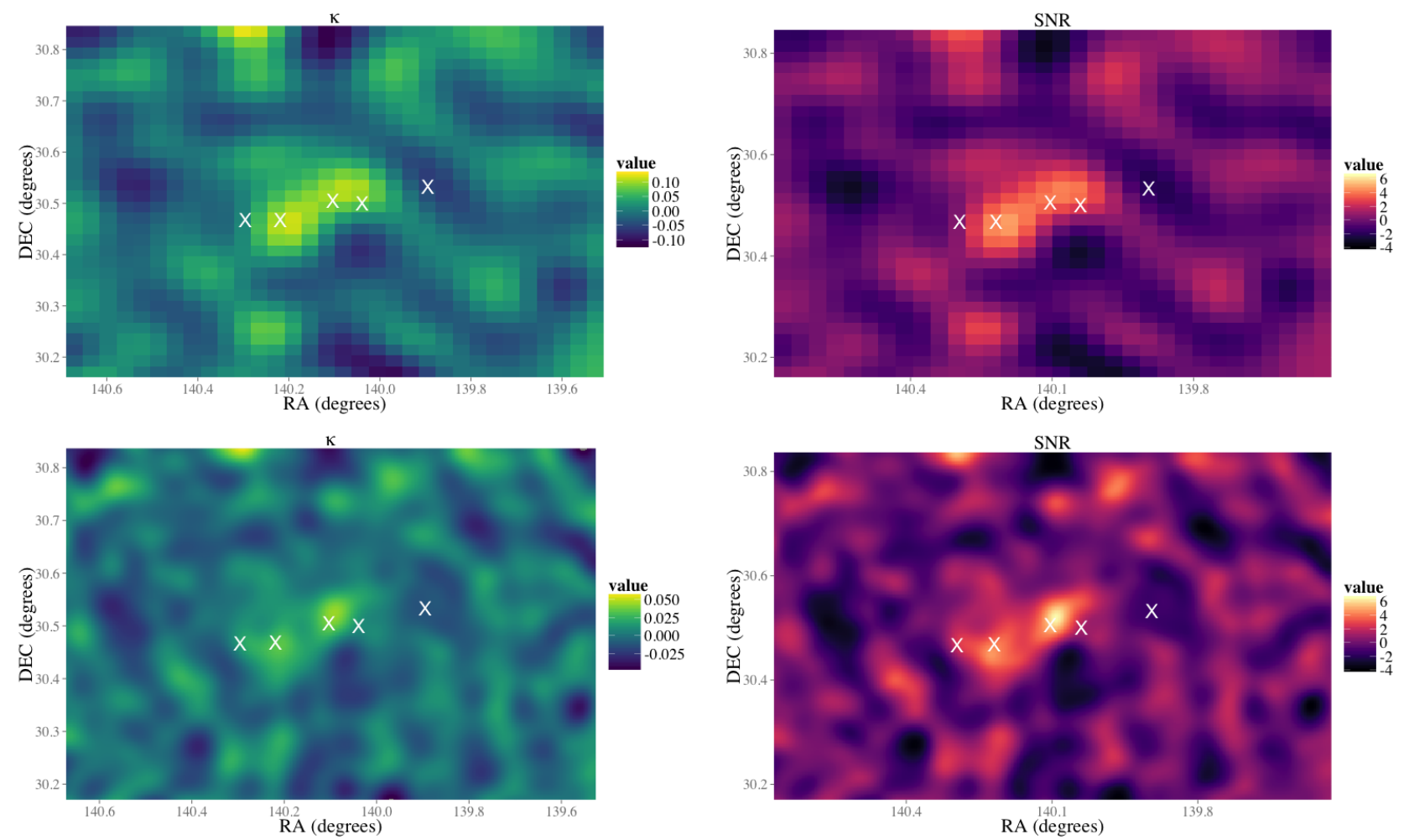

FIG. 10.- Posterior mean lens field maps (left) and SNR maps (right) for a field centered on the galaxy cluster Abell 781 . We use 6000 galaxies selected to have photometric redshifts larger than the known redshifts of the two primary clusters in this field of view $(z=0.296$ for Abell 781 and $z=0.43$ for a chance alignment of a second cluster).The top row of panels show the posterior mean map and SNR using our GP prior. The bottom row of panels show the algorithm of Dawson et al. (2012) applied to the same galaxies with the same uniform per-galaxy weighting as in the top row, but evaluated on a finer grid. The normalization of the convergence in the lower left panel is arbitrary (see Dawson et al. 2012). The white crosses indicate sub-clusters identified by Sehgal et al. (2008) with x-ray detections. There is no associated mass for the white cross second from the right in any published analyses of this system.

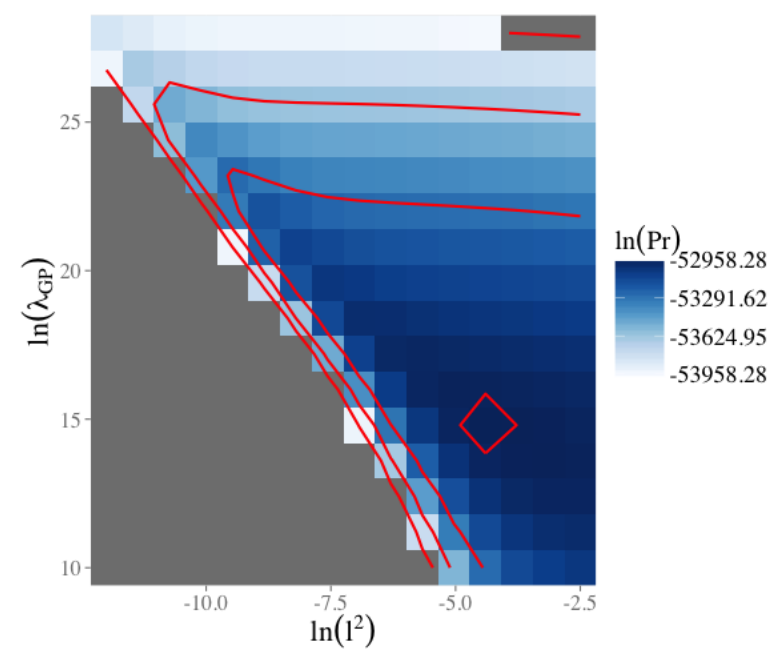

FIG. 11. - The marginal posterior distribution for the GP parameters with priors imposed to favor correlation lengths smaller than the expected cluster sizes.

number of galaxies. Next generation surveys will have $10^{7}-10^{10}$ galaxies, requiring both parallelization of the linear solver routines and optimizations such as sparse matrix approximations via tapering (Kaufman 2008) or Fast Fourier Transform (FFT)-based matrix multiplications (exploiting the special structure of the isotropic cosmological covariances Padmanabhan et al. 2003). As a next step in the validation and scaling of our code we plan to apply the algorithms in this paper to the variable shear 'branches' of the GREAT3 challenge ${ }^{11}$ (Mandelbaum et al. 2014), which contain $2 \times 10^{5}$ galaxies per simulated field. Coincidentally, each of the five DLS four square degree fields contains a comparable number of $\sim 2 \times 10^{5}$ lensing source galaxies.

For the algorithm we validated in this paper we assumed an approximate data vector of galaxy ellipticity measurements rather than the more informative imaging pixel data for each galaxy, as we described previously in Schneider et al. (2015). If we use galaxy samples rather than an ellipticity catalog then we have to go back to Equation (1) and replace the integral over the intrinsic ellipticities with a numerical importance sampling formula from Schneider et al. (2015). In this case we do not need to assume conjugacy of the intrinisc ellipticity prior and the likelihood function, which is good because the pixel-level likelihood will not be conjugate. The algorithms we presented in subsubsection 3.1.1 for cosmological parameter estimation are applicable, however, when using either an approximate ellipticity data vector or the interim sampling of galaxy image model parameters.

We can still derive a Wiener Filter from the product of the Gaussian intrinsic ellipticity distribution (or DP base distribution) and the interim variable shear GP prior assuming a weak shear approximation. But then we need to evaluate Equation (15) and Equation (16) for every interim sample. The parameters of $\mathrm{S}+\mathrm{N}$ include the GP

\footnotetext{
11 http://great3challenge.info/
} 
parameters in $\mathrm{S}$ and the $\alpha$ intrinsic ellipticity distribution parameter in $\mathrm{N}$. If we update either parameter we need to perform a new matrix factorization and solve operation at every step (but these operations can be done once for all interim samples of galaxy image model parameters in a given step). Exploring optimized linear algebra approaches and effective sampling strategies for this more complete framework will be a focus of future work.

We have not included any redshift information about the source galaxies in our model. However, the use of such information (via photometric redshifts) is a critical component of the weak lensing analyses for cosmic shear surveys as well as cluster mass reconstructions with optimized signal-to-noise ratios. A simple extension of our work to include redshifts could be to impose separable GP priors on the lens fields for galaxy samples binned in redshift (or redshift estimator). The physical correlations between the lens fields inferred in each source bin can be modeled in the hierarchical inference stage when marginalizing lens fields to infer cosmological parameters (as outlined in Schneider et al. 2015). A more thorough approach would be to include probabilistic redshifts for each source galaxy and marginalize over each source redshift distribution as part of the lens field inference.

\section{ACKNOWLEDGMENTS}

We thank Chris Paciorek for discussion about the statistical framework for performing shear inference and the use of Gaussian Processes. We thank Alex Malz for critical reviews of draft versions of this work. Thanks to M. James Jee for providing the Deep Lens Survey shear catalog. Part of this work performed under the auspices of the U.S. Department of Energy by Lawrence Livermore National Laboratory under Contract DE-AC5207NA27344. Funding for this work was provided by LLNL Laboratory Directed Research and Development grant 16-ERD-013. This work was also supported by the Director, Office of Science, Office of Advanced Scientific Computing Research, Applied Mathematics program of the U.S. Department of Energy under Contract No.DEAC02-05CH11231. This research used resources of the National Energy Research Scientific Computing Center (NERSC), a DOE Office of Science User Facility supported by the Office of Science of the U.S. Department of Energy under Contract No. DE-AC02-05CH11231. This work uses a modified version of the public code George available at https://github.com/karenyyng/ george, which was forked from https://github.com/ $\mathrm{dfm} /$ george. We also made use of the daft package (developed by Dan Foreman-Mackey, David W. Hogg, and contributors, and available at http://daft-pgm.org/) for plotting probabilistic graphical models.

\section{REFERENCES}

Alsing, J., Heavens, A., Jaffe, A. H., et al. 2015, 16 [1, 2.1]

Alsing, J., Heavens, A. F., \& Jaffe, A. H. 2016, ArXiv e-prints [1]

Amara, A., Lilly, S., Kovač, K., et al. 2012, MNRAS, 424, 553 [1]

Ambikasaran, S. 2013, A fast direct solver for dense linear systems, https://github.com/sivaramambikasaran/HODLR [A]

Bard, D., Kratochvil, J. M., \& Dawson, W. 2016, ApJ, 819, 158 [1]

Bartelmann, M. 1995, A\&A, 303, 643 [1]

Bartelmann, M., Narayan, R., Seitz, S., \& Schneider, P. 1996, ApJ, 464, L115 [1]

Bartelmann, M. \& Schneider, P. 2001, Phys. Rep., 340, 291 [2]

Bridle, S. L., Hobson, M. P., Lasenby, A. N., \& Saunders, R. 1998, Monthly Notices of the Royal Astronomical Society, 299, $895[1]$

Bridle, S. L., Hobson, M. P., Saunders, R., \& Lasenby, A. N. 2000, ArXiv Astrophysics e-prints [1]

Bunn, E. F., Zaldarriaga, M., Tegmark, M., \& de Oliveira-Costa, A. 2003, Phys. Rev. D, 67, 023501 [3.1, 6]

Carron, J. 2014, ArXiv e-prints [1]

Chang, C., Vikram, V., Jain, B., et al. 2015, Physical Review Letters, 115, 051301 [1]

Cooray, A. \& Hu, W. 2001, ApJ, 554, 56 [3.1.1]

Cottle, R. W. 1974, Linear Algebra and its Applications, 8, 189 $[\mathrm{C}]$

Dawson, W. A., Wittman, D., Jee, M. J., et al. 2012, ApJ, 747, L42 $[3.2,10]$

Deriaz, E., Starck, J.-L., \& Pires, S. 2012, A\&A, 540, A34 [1]

Dietrich, J. P., Schneider, P., Clowe, D., Romano-Díaz, E., \& Kerp, J. 2005, A\&A, 440, 453 [1]

Dodelson, S. 2003, Modern Cosmology, Academic Press (Academic Press) []

Elsner, F. \& Wandelt, B. D. 2013, A\&A, 549, A111 [1]

Gray, M. E., Taylor, A. N., Meisenheimer, K., et al. 2002, ApJ, $568,141[1]$

Hamilton, A. J. S. 2000, MNRAS, 312, 257 [13]

Hamilton, A. J. S., Rimes, C. D., \& Scoccimarro, R. 2006, 371, $1188[1]$

Heymans, C., Gray, M. E., Peng, C. Y., et al. 2008, MNRAS, $385,1431[1]$

Jasche, J. \& Wandelt, B. D. 2013, MNRAS, 432, 894 [2.2]

Jaynes, E. T. 1957, Phys. Rev., 106, 620 [2.1]
Jee, M. J., Tyson, J. A., Hilbert, S., et al. 2016, ApJ, 3962 [1, 3.2] Jee, M. J., Tyson, J. A., Schneider, M. D., et al. 2013, ApJ, 765, $74[3,3.2]$

Jiao, Y.-X., Shan, H.-Y., \& Fan, Z.-H. 2011, Research in Astronomy and Astrophysics, 11, 507 [1]

Joachimi, B., Schneider, P., \& Eifler, T. 2008, A\&A, 477, 43 [B]

Kacprzak, T., Zuntz, J., Rowe, B., et al. 2012, MNRAS, 427, 2711 $[2]$

Kaiser, N. \& Squires, G. 1993, ApJ, 404, 441 [1]

Kamionkowski, M., Kosowsky, A., \& Stebbins, A. 1997, Phys. Rev. D, 55, 7368 [B]

Kaufman, C. 2008, Journal of the American Statistical Association [4]

Kratochvil, J. M., Haiman, Z., \& May, M. 2010, Phys. Rev. D, 81,043519 [1]

Kubo, J. M., Khiabanian, H., Dell'Antonio, I. P., Wittman, D., \& Tyson, J. A. 2009, ApJ, 702, 980 [1, 3.2]

Liu, J. \& Haiman, Z. 2016, Phys. Rev. D, 94, 043533 [1]

Liu, J., Petri, A., Haiman, Z., et al. 2015, Phys. Rev. D, 91, $063507[1]$

Liu, X., Li, B., Zhao, G.-B., et al. 2016, Phys. Rev. Lett., 117, $051101[1]$

Liu, X., Wang, Q., Pan, C., \& Fan, Z. 2014, The Astrophysical Journal, 784,31 [1]

Lombardi, M. \& Bertin, G. 1998, A\&A, 335, 1 [1]

Mandelbaum, R., Rowe, B., Armstrong, R., et al. 2015, MNRAS, 450, $2963[3.2]$

Mandelbaum, R., Rowe, B., Bosch, J., et al. 2014, ApJS, 212, 5 [4]

Marshall, P. J., Hobson, M. P., Gull, S. F., \& Bridle, S. L. 2001 Mon. Not. R. Astron. Soc., 335, 1037. 12 p [1, 2.1.1]

Marshall, P. J., Hobson, M. P., Gull, S. F., \& Bridle, S. L. 2013, LensEnt2: Maximum-entropy weak lens reconstruction, Astrophysics Source Code Library [1]

Massey, R., Rhodes, J., Ellis, R., et al. 2007, Nature, 445, 286 [1]

Morrison, C. B. \& Schneider, M. D. 2013, JCAP, 11, 9 [1]

Ng, K. Y. 2016, PhD thesis, University of California, Davis [2.1, A]

Padmanabhan, N., Seljak, U., \& Pen, U. L. 2003, New Astron., 8, 581 [4]

Pan, J. \& Szapudi, I. 2005, 361, 357 [1] 
Petri, A., May, M., \& Haiman, Z. 2016, ArXiv e-prints [1] Racine, B., Jewell, J. B., Eriksen, H. K., \& Wehus, I. K. 2016, ApJ, 820, 31 [1]

Refregier, A., Kacprzak, T., Amara, A., Bridle, S., \& Rowe, B. 2012, MNRAS, 425, 1951 [2]

Reischke, R., Maturi, M., \& Bartelmann, M. 2016, MNRAS, 456, 641 [1]

Rowe, B. T. P., Jarvis, M., Mandelbaum, R., et al. 2015, Astronomy and Computing, 10, 121 [2]

Schmidt, F. \& Rozo, E. 2011, ApJ, 735, 119 [1]

Schmidt, S. J. \& Thorman, P. 2013, MNRAS, 431, 2766 [3.2]

Schneider, M. D., Hogg, D. W., Marshall, P. J., et al. 2015, ApJ, $807,87[1,2,2,1,2,4]$

Schneider, P. \& Seitz, C. 1995, A\&A, 294, 411 [1]

Schneider, P., van Waerbeke, L., Kilbinger, M., \& Mellier, Y. 2002a, A\&A, 396, 1 [B]

Schneider, P., van Waerbeke, L., \& Mellier, Y. 2002b, A\&A, 389, 729 [B]

Sehgal, N., Hughes, J. P., Wittman, D., et al. 2008, The Astrophysical Journal, 673, 163 [3.2, 10]

Seitz, S., Schneider, P., \& Bartelmann, M. 1998, A\&A, 337, 325 [1]

Shan, H. Y., Kneib, J.-P., Comparat, J., et al. 2014, MNRAS, $442,2534[1,3.1]$

Shore, J. \& Johnson, R. 1980, Information Theory, IEEE Transactions on, 26, 26 [2.1]

Starck, J.-L., Murtagh, F., Querre, P., \& Bonnarel, F. 2001, A\&A, 368, 730 [1]
Starck, J.-L., Pires, S., \& Réfrégier, A. 2006, A\&A, 451, 1139 [1]

Takada, M. \& Hu, W. 2013, arXiv.org [1]

Tyson, J. A., Valdes, F., \& Wenk, R. A. 1990, ApJ, 349, L1 [1] van Waerbeke, L. 2000, MNRAS, 313, 524 [1, 3.1]

Van Waerbeke, L., Benjamin, J., Erben, T., et al. 2013, MNRAS, $433,3373[1]$

Vasicek, O. 1976, Journal of the Royal Statistical Society. Series B (Methodological), 38, 54 [2.1]

Vikram, V., Chang, C., Jain, B., et al. 2015, Phys. Rev. D, 92, 022006 [1]

Wandelt, B. D., Larson, D. L., \& Lakshminarayanan, A. 2004 , Phys. Rev. D, 70, 083511 [1]

Wiener, N. 1949, Extrapolation, interpolation, and smoothing of stationary time series, Vol. 2 (MIT press Cambridge) [1]

Wittman, D., Dawson, W., \& Benson, B. 2014, MNRAS, 437, $3578 \quad[3.2,3.2]$

Wittman, D., Dell'Antonio, I. P., Hughes, J. P., et al. 2006, ApJ, $643,128 \quad[3.2]$

Wittman, D. M., Tyson, J. A., Dell'Antonio, I. P., et al. 2002, in Society of Photo-Optical Instrumentation Engineers (SPIE) Conference Series, Vol. 4836, Survey and Other Telescope Technologies and Discoveries, ed. J. A. Tyson \& S. Wolff, 73-82 $[3,3.2]$

\section{APPENDIX}

\section{GAUSSIAN PROCESS COVARIANCES}

We use throughout our analysis the the squared exponential kernel for the GP prior on the lens potential as specified in Equation (12). A squared exponential kernel only depends on the distances between pairs of galaxy locations. This chosen form is invariant under translational and rotational transformations (homogeneous and isotropic). Compared with other forms of isotropic covariance kernels, the exponential squared kernel produces spatial fields that are smoother for the same correlation length parametrization $\ell_{\mathrm{GP}}^{2}=\exp \left(-1 /\left(8 \ln \rho_{\mathrm{GP}}\right)\right)$, where $\rho_{\mathrm{GP}}$ is a correlation coefficient on the interval $[0,1]$ provided the locations $\mathbf{x}$ are normalized to the unit interval. Furthermore, the exponential squared kernel is infinitely differentiable. This infinitely differentiable kernel choice allows us to derive an analytical expression to relate different lensing observables through differentiation.

Since the covariance operator is linear, the derivatives for the covariance kernels of interests are:

$$
\begin{aligned}
\operatorname{Cov}(\kappa(\mathbf{x}), \kappa(\mathbf{y})) & =\frac{1}{4}\left(\mathrm{~S}_{, 1111}+\mathrm{S}_{, 1122}+\mathrm{S}_{, 2211}+\mathrm{S}_{, 2222}\right) \\
\operatorname{Cov}\left(\kappa(\mathbf{x}), \gamma_{1}(\mathbf{y})\right) & =\frac{1}{4}\left(\mathrm{~S}_{, 1111}+\mathrm{S}_{, 2211}-\mathrm{S}_{, 1122}-\mathrm{S}_{, 2222}\right) \\
\operatorname{Cov}\left(\kappa(\mathbf{x}), \gamma_{2}(\mathbf{y})\right) & =\frac{1}{4}\left(\mathrm{~S}_{, 1112}+\mathrm{S}_{, 2212}+\mathrm{S}_{, 1121}+\mathrm{S}_{, 2221}\right) \\
\operatorname{Cov}\left(\gamma_{1}(\mathbf{x}), \gamma_{1}(\mathbf{y})\right) & =\frac{1}{4}\left(\mathrm{~S}_{, 1111}-\mathrm{S}_{, 1122}-\mathrm{S}_{, 2211}+\mathrm{S}_{, 2222}\right) \\
\operatorname{Cov}\left(\gamma_{1}(\mathbf{x}), \gamma_{2}(\mathbf{y})\right) & =\frac{1}{4}\left(\mathrm{~S}_{, 1112}+\mathrm{S}_{, 1121}-\mathrm{S}_{, 2212}-\mathrm{S}_{, 2221}\right) \\
\operatorname{Cov}\left(\gamma_{2}(\mathbf{x}), \gamma_{2}(\mathbf{y})\right) & =\frac{1}{4}\left(\mathrm{~S}_{, 1212}+\mathrm{S}_{, 1221}+\mathrm{S}_{, 2112}+\mathrm{S}_{, 2121}\right)
\end{aligned}
$$

where

$$
\mathrm{S}_{, h i j k}=\frac{\partial^{4} \mathrm{~S}}{\partial x_{h} \partial x_{i} \partial y_{j} \partial y_{k}},
$$

and we have defined the shorthand for spatial derivatives with subscripts for $h, i, j, k=1,2$ after a comma.

Generalizing the definition of $s^{2}$ from Equation (13) to include an arbitrary distance metric,

$$
s^{2}=(\mathbf{x}-\mathbf{y})^{T} \mathrm{D}(\mathbf{x}-\mathbf{y}),
$$

and with the definition of $\chi_{i}$ as follows:

$$
\frac{\partial s^{2}}{\partial \mathbf{x}_{i}}=-\frac{\partial s^{2}}{\partial \mathbf{y}_{i}}=2 \mathrm{D}(\mathbf{x}-\mathbf{y})_{i} \equiv 2 \chi_{i},
$$


we can show that each entry $\nu_{, h i j k}$ of $S_{, h i j k}$ is related to each entry $\nu$ of the original exponential squared kernel $S$ by:

$$
\nu_{, x_{h} x_{i} y_{j} y_{k}}=\left(\beta^{4} \chi_{h} \chi_{i} \chi_{j} \chi_{k}-\beta^{3}\left(\chi_{h} \chi_{i} \mathrm{D}_{j k} \delta_{j k}+5 \text { perm. }\right)+\beta^{2}\left(\mathrm{D}_{h j} \mathrm{D}_{i k} \delta_{h j} \delta_{i k}+2 \text { perm. }\right)\right) \nu,
$$

with $\beta \equiv-1 /\left(8 \ln \rho_{\mathrm{GP}}\right)=\ln \left(\ell_{\mathrm{GP}}^{2}\right)$. There are 6 permutations (abbreviated as perm.) of the terms in A10 multiplied by $\beta^{3}$ due to choosing two pairs of indices from the $h, i, j, k$, where the order of each pair of indices matters. Likewise, there are 3 possible permutations of the terms multiplied by $\beta^{2}$ due to choosing two pairs of indices from $h, i, j, k$, where the order of the pairs does not matter. The full derivation of the relevant derivatives and terms can be found in $\mathrm{Ng}$ (2016). The full covariance model is implemented in a fork of the public GP code George ${ }^{12}$ (Ambikasaran 2013 ).

We show the structure of the GP covariance matrix, S, from Equation (12) in Figure 12 for a simulation with 1600 galaxies placed on a uniform $40 \times 40$ grid on the sky and a coarser grid of $24 \times 24$ locations to which the lens fields are interpolated. The distinctive banded structure is because of the galaxy locations on a uniform $2 \mathrm{D}$ grid that is then flattened into a 1D array. The sub-blocks correspond to the $\kappa, \gamma_{1}, \gamma_{2}$ fields for the grid and galaxy locations. A notable feature of the covariance in Figure 12 is the rapid decrease in absolute values of the elements moving away from the diagonals of each sub-block. We will explore in future work whether sparse linear algebra software packages may be useful for speeding up the computations in our lens field inference.

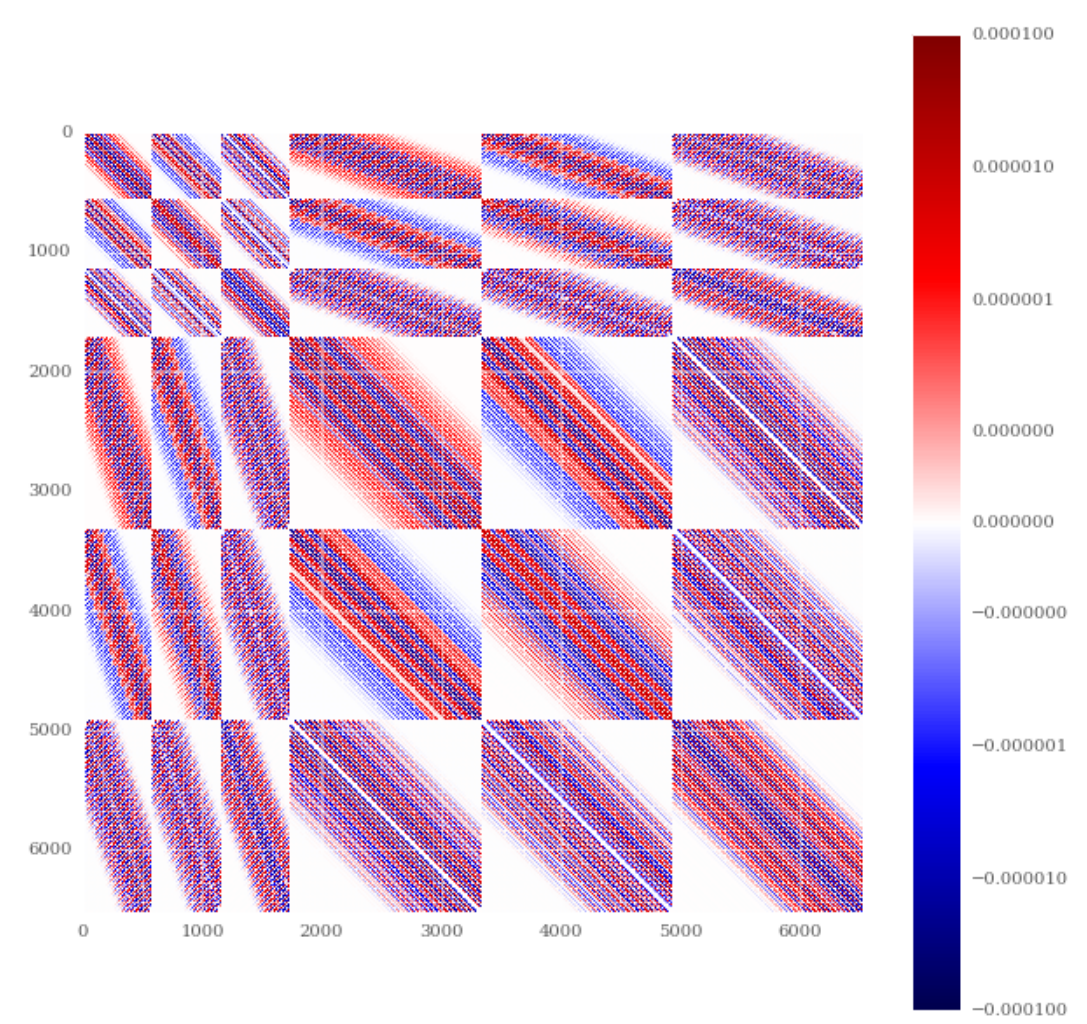

FIG. 12.- The Gaussian Process covariance used in our simulation validation study. This covariance matches that used for Figure 4, corresponding to $\sigma_{e}=0.0026$. There are 1600 galaxy locations, 576 interpolation grid locations, and 3 lens fields for a total covariance dimension of $6528^{2}$. There are 4 large sub-blocks per dimension. The first large sub-block represents the grid locations to which we interpolate from the galaxy locations. The 3 remaining large sub-blocks represent $\kappa, \gamma_{1}, \gamma_{2}$ at the galaxy locations. Inside the first large sub-block is the $\kappa, \gamma_{1}, \gamma_{2}$ sub-sub-blocks for the grid locations.

It is possible to generalize the derivation of derivatives by using numerical differentiation. Numerical differentiation may allow evaluation of other isotropic kernels, such as the Matérn kernels. An implementation via numerical differentiation may or may not be more computationally intensive to compute, depending on the required numerical precision (aka order of accuracy). Alternative differentiation technology such as automatic differentiation can be considered but is outside the scope of this paper.

\section{COSMOLOGY-DEPENDENT COVARIANCE MODEL}

Under linear cosmological perturbation theory, we can model the lensing convergence as Gaussian distributed with a specified isotropic angular power spectrum. This power spectrum then defines a cosmological covariance model for the lens fields, which we derive and explore in this section.

Gravitational lensing generates only E-mode shear distortions for a uniform distribution of source illumination. Clustered sources can generate B-mode shear, but only on sub-arcminute scales for typical cosmological models (Schneider

12 https://github.com/dfm/george

https://github.com/karenyyng/george 
et al. 2002b). We consider then a real-valued lensing convergence $\kappa=\kappa_{E}$ with an associated angular power spectrum $P_{E}(\ell)$, under a flat-sky approximation. In the left panel of Figure 13 we show the angular convergence power spectrum used in our simulation study from subsection 3.1.

The covariance of shear and convergence is most easily described in terms of the rotated shear components (Schneider et al. 2002a; Joachimi et al. 2008),

$$
\gamma_{t} \equiv-\operatorname{Re}\left(\gamma e^{-2 i \phi}\right) \quad \gamma_{\times} \equiv-\operatorname{Im}\left(\gamma e^{-2 i \phi}\right)
$$

where $\phi$ is the polar angle of the angular separation vector of a galaxy pair. In the flat-sky limit, the two-point functions of the convergence and shear components are then related to the convergence power spectrum as (in analogy with CMB polarization correlation functions Kamionkowski et al. 1997),

$$
\begin{aligned}
\left\langle\kappa_{E} \kappa_{E}\right\rangle(|\mathbf{x}|) & =\int_{0}^{\infty} \frac{\ell d \ell}{2 \pi} P_{E}(\ell) J_{0}(\ell|\mathbf{x}|) \\
\left\langle\kappa_{E} \gamma_{t}\right\rangle(|\mathbf{x}|) & =\int_{0}^{\infty} \frac{\ell d \ell}{2 \pi} P_{E}(\ell) J_{2}(\ell|\mathbf{x}|) \\
\left\langle\gamma_{t} \gamma_{t}\right\rangle(|\mathbf{x}|) & =\frac{1}{2} \int_{0}^{\infty} \frac{\ell d \ell}{2 \pi} P_{E}(\ell)\left[J_{0}(\ell|\mathbf{x}|)+J_{4}(\ell|\mathbf{x}|)\right] \\
\left\langle\gamma_{\times} \gamma_{\times}\right\rangle(|\mathbf{x}|) & =\frac{1}{2} \int_{0}^{\infty} \frac{\ell d \ell}{2 \pi} P_{E}(\ell)\left[J_{0}(\ell|\mathbf{x}|)-J_{4}(\ell|\mathbf{x}|)\right],
\end{aligned}
$$

where $J_{n}$ is the $n$-th order Bessel function of the first kind. We can recover the correlation functions of the shear components measured with respect to a fixed sky coordinate system from,

$$
\begin{aligned}
& \gamma_{1}=-\cos (2 \phi) \gamma_{t}+\sin (2 \phi) \gamma_{\times} \\
& \gamma_{2}=-\sin (2 \phi) \gamma_{t}-\cos (2 \phi) \gamma_{\times} .
\end{aligned}
$$

Then,

$$
\begin{aligned}
& \left\langle\gamma_{1} \gamma_{1}\right\rangle=\cos ^{2}(2 \phi)\left\langle\gamma_{t} \gamma_{t}\right\rangle+\sin ^{2}(2 \phi)\left\langle\gamma_{\times} \gamma_{\times}\right\rangle \\
& \left\langle\gamma_{2} \gamma_{2}\right\rangle=\sin ^{2}(2 \phi)\left\langle\gamma_{t} \gamma_{t}\right\rangle+\cos ^{2}(2 \phi)\left\langle\gamma_{\times} \gamma_{\times}\right\rangle \\
& \left\langle\gamma_{1} \gamma_{2}\right\rangle=\sin (2 \phi) \cos (2 \phi)\left(\left\langle\gamma_{t} \gamma_{t}\right\rangle-\left\langle\gamma_{\times} \gamma_{\times}\right\rangle\right) .
\end{aligned}
$$

We show the angular correlation functions contributing to the convergence and shear auto- and cross-correlations in the right panel of Figure 13. Specifically we plot,

$$
\xi_{n}(\theta) \equiv \int \frac{\ell d \ell}{2 \pi} P_{E}(\ell) J_{n}(\ell \theta) .
$$
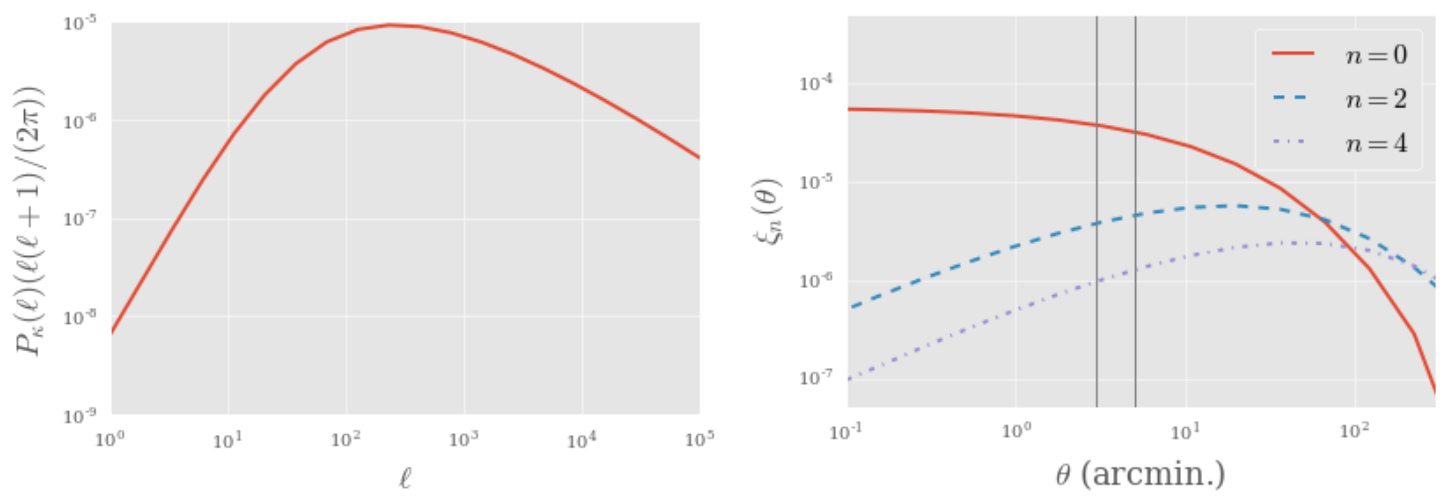

FIG. 13.- Left: The convergence power spectrum used to simulate shear fields in our simulation study in subsection 3.1. The power spectrum is calculated for linear cosmological perturbations with a source distribution narrowly peaked at $z=1$. Right: The angular correlation functions obtained by integrating the power spectrum from the left panel with different Bessel function weights. We perform the Bessel function integrals using the Hankel transforms implemented in FFTLOG (Hamilton 2000). The vertical lines denote the grid sizes for the input galaxies and the interpolation grid in subsection 3.1. Angular scales to the left of these lines are unresolved in our simulation grids.

We show the resulting joint covariance of convergence and shear in Figure 14. The covariance is evaluated at the same sky locations as in Figure 12. It is notable that the covariance in Figure 14 has larger relative amplitudes between the diagonal and off-diagonal components of each sub-block than in Figure 12. This indicates that the effective kernel for averaging shears to infer the convergence is broader under this cosmological model than in the covariance we 
determined under the GP model. Our GP model is thus discarding more information from distant galaxies in inferring the convergence at any given sky location.

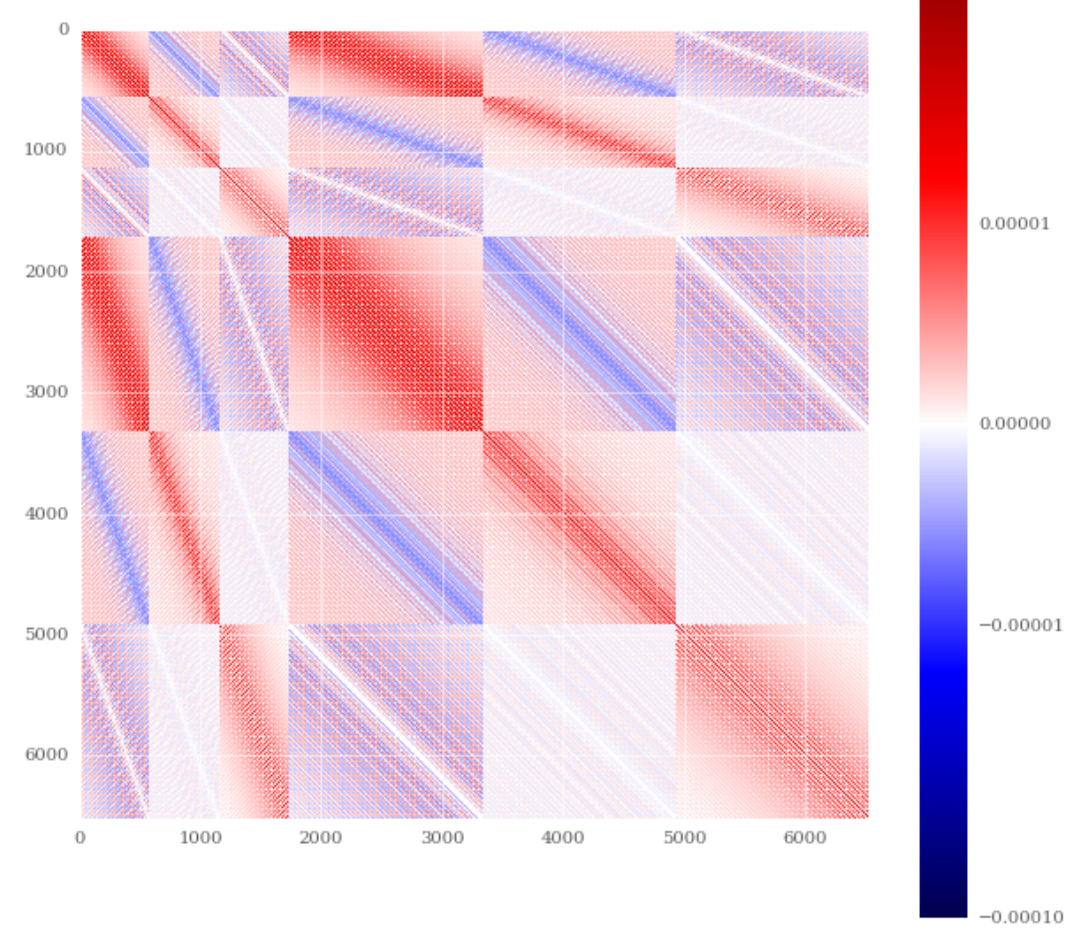

FIG. 14.- The covariance of convergence and shear derived from the cosmological power spectrum used in our simulation validation study.

To further understand the relative performance of the cosmological and GP model covariances for lens field mapping, in Figure 15 we reproduce the maps shown in Figure 4 but now using the cosmology covariance instead of the GP kernel. The reconstruction of all lens fields in the left panel of Figure 15 is superb given the different input and interpolated grid sizes. This is expected because the input data is strictly Gaussian distributed and we used the same power spectrum to simulate the data and to perform the WF interpolation. However, comparing the right panel of Figure 15 with that of Figure 4 shows the SNR of the mean posterior maps using the cosmology covariance is smaller than that using the GP covariance. This is because the WF variance using the cosmology covariance is larger than that using the GP covariance.

In Figure 16 we show the E and B mode power spectra derived from the maps in Figure 15. The E-mode power spectrum reconstruction is more accurate at frequencies closer to Nyquist than that in Figure 5 derived from the GP model. However, the B-mode power spectrum amplitude is larger in Figure 16 than in Figure 5. We show the corresponding $\mathrm{E}$ and $\mathrm{B}$ mode maps derived from the mean posterior using the cosmology covariance in Figure 17. The E-mode map in Figure 17 is an even better match to the input convergence field than the posterior convergence map in Figure 15. This indicates there is some level of B-mode leakage in the posterior convergence map in Figure 15. The B-mode map in Figure 17 has non-negligible amplitude (consistent with Figure 16) and contains more spatial structure than the B-mode map in Figure 6 derived from the GP prior.

In summary, the comparison of the mean posterior lens fields and corresponding power spectra between the GP and cosmological covariance models shows the GP covariance yields higher SNR mass map extrema and smaller B-mode power at the expense of reduced dynamic range in the power spectrum relative to the cosmological model covariance (all conditioned on the particular choice of GP parameters).

\section{LINEAR THEORY COSMOLOGICAL PARAMETER DEPENDENCE}

In this section we derive the form of the covariance that appears in the marginal likelihood for galaxy ellipticities when assuming linear theory, weak shear, and a Gaussian likelihood as in Equation (34).

The marginal likelihood requires evaluation of Equation (33), which has as left-hand side,

$$
\operatorname{Pr}\left(\Upsilon \mid \Upsilon^{\prime}, a_{\mathrm{GP}}\right) \operatorname{Pr}\left(\Upsilon^{\prime} \mid \theta\right)
$$

Each term in Equation C1 is a Normal distribution, so evaluating the product in Equation C1 reduces to evaluating 

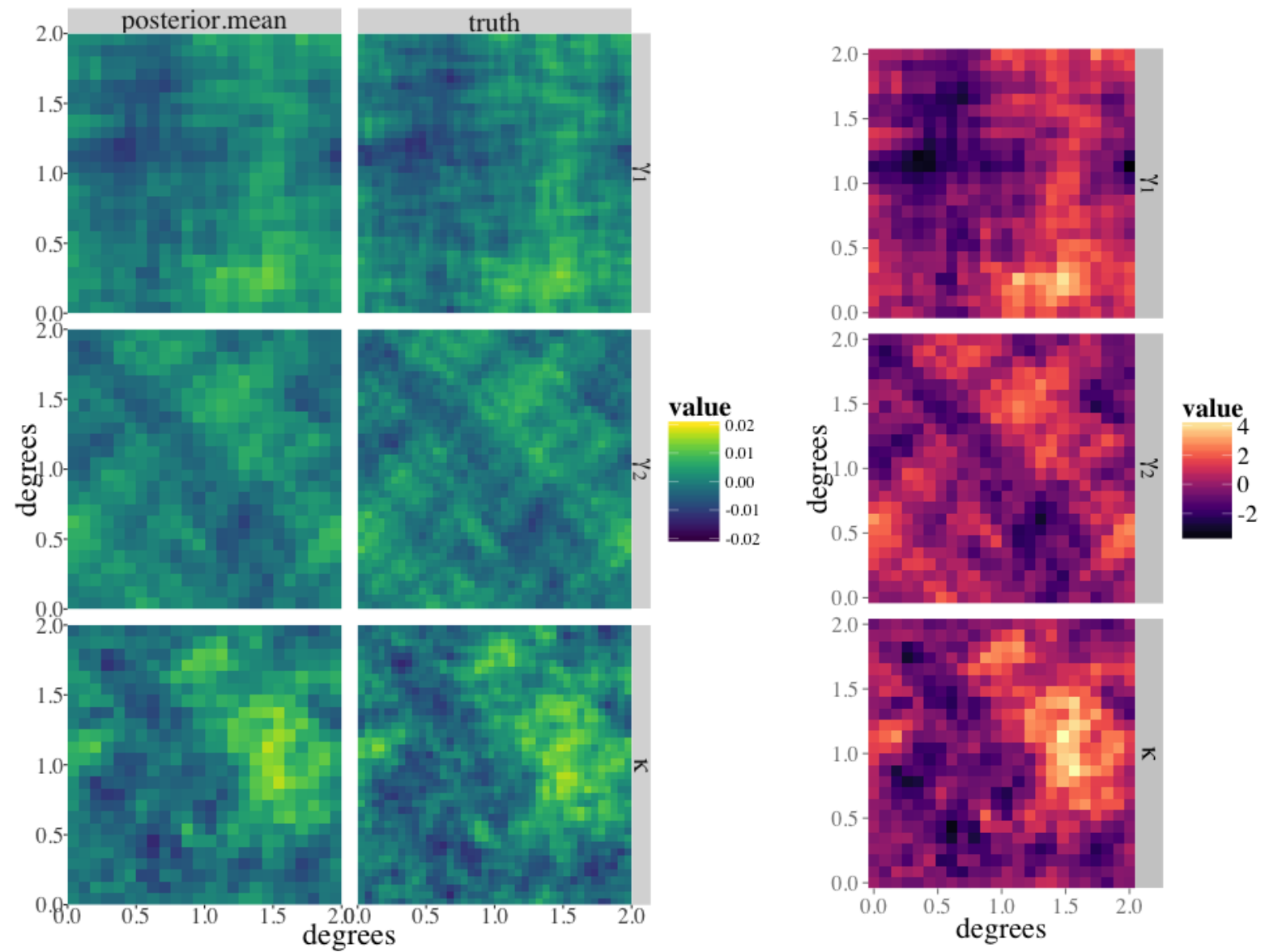

FIG. 15.- Left: Comparison of the convergence and shear maps in our simulation study between that used to generate our mock galaxy ellipticity catalog (right column) and the output of our GP interpolation (left column). The rows show the maps for the two shear components $\gamma_{1,2}$ and the convergence $\kappa$. These maps cover a $2 \times 2$ square degree field. The simulated intrinsic ellipticity r.m.s. is set to an artificially small value of $\sigma_{e}=0.0026$, which is 100 times smaller than that observed for the complete Deep Lens Survey catalog. Right: signal-to-noise ratio (SNR) maps for the same simulations. We calculate SNR as the ratio of the absolute value of the map to the square root of the diagonal of the covariance in Equation (15). These simulations use 1600 galaxies and grid sizes for the GP interpolated shear and convergence with 24 grid cells per dimension.

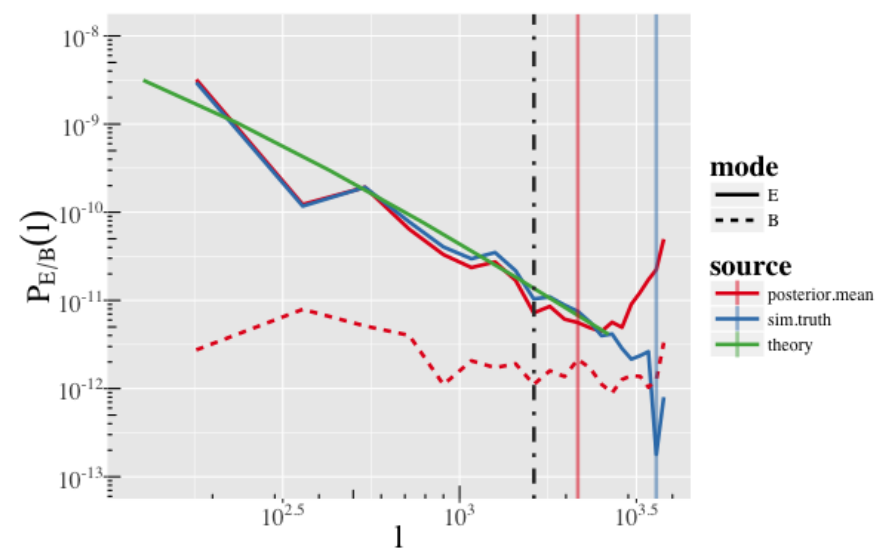

FIG. 16. - Comparison of power spectrum estimators in our simulation study to the 'truth' input spectrum. The vertical solid lines show the Nyquist frequencies of the grids. The vertical dot-dashed matches that in Figure 5 to aide comparison of the figures, but has no relationship to the plotted power spectra here.

the sum of the inverse covariances of each term. It is convenient to evaluate the identical expression to Equation C1,

$$
\operatorname{Pr}\left(\Upsilon \mid \Upsilon^{\prime}, a_{\mathrm{GP}}\right) \operatorname{Pr}\left(\Upsilon^{\prime} \mid \theta\right)=\operatorname{Pr}\left(\Upsilon \mid \Upsilon^{\prime}, a_{\mathrm{GP}}\right) \operatorname{Pr}\left(\Upsilon^{\prime} \mid a_{\mathrm{GP}}\right) \frac{\operatorname{Pr}\left(\Upsilon^{\prime} \mid \theta\right)}{\operatorname{Pr}\left(\Upsilon^{\prime} \mid a_{\mathrm{GP}}\right)} .
$$




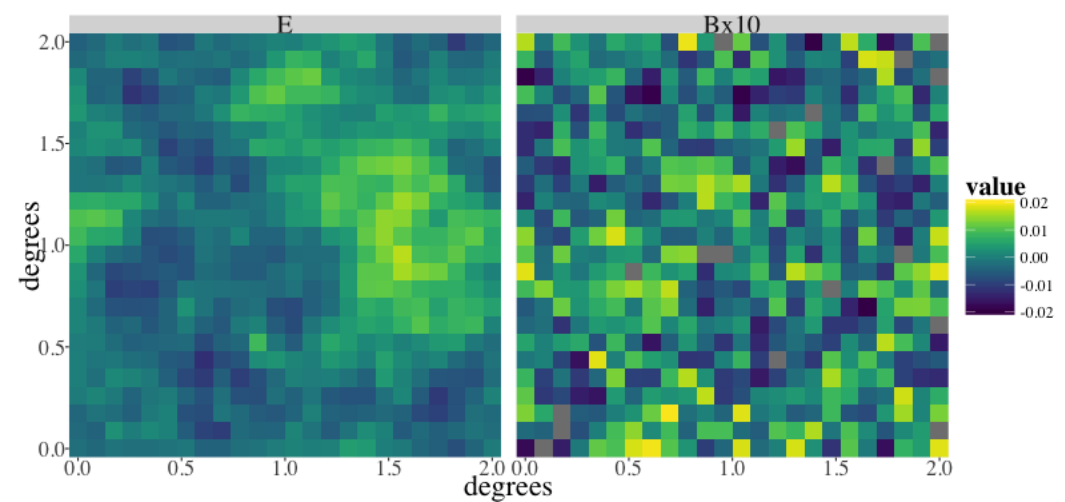

FIG. 17.- E and B mode maps derived from the posterior mean shear maps shown in the left column of Figure 15. Note the value of the plotted B-mode map has been multiplied by 10 for easier visualization.

Next, factor the GP covariance in $\operatorname{Pr}\left(\Upsilon \mid \Upsilon^{\prime}, a_{\mathrm{GP}}\right) \operatorname{Pr}\left(\Upsilon^{\prime} \mid a_{\mathrm{GP}}\right)$ into blocks that depend on coordinates $\mathbf{x}$ and $\mathbf{x}^{\prime}$,

$$
\mathrm{S}_{\mathrm{GP}}=\left(\begin{array}{cc}
\mathrm{S}_{\mathrm{GP}}(\mathbf{x}, \mathbf{x}) & \mathrm{S}_{\mathrm{GP}}\left(\mathbf{x}, \mathbf{x}^{\prime}\right) \\
\mathrm{S}_{\mathrm{GP}}\left(\mathbf{x}^{\prime}, \mathbf{x}\right) & \mathrm{S}_{\mathrm{GP}}\left(\mathbf{x}^{\prime}, \mathbf{x}^{\prime}\right)
\end{array}\right) \equiv\left(\begin{array}{cc}
\mathrm{A} & \mathrm{B} \\
\mathrm{B}^{T} & \mathrm{C}
\end{array}\right)
$$

where the second equality defines a short-hand notation for the matrix blocks. We can invert Equation C3 using the expressions for the conditional covariance, or Schur complement (e.g., Cottle 1974),

$$
\mathrm{S}_{\mathrm{GP}}^{-1}=\left(\begin{array}{cc}
\mathrm{W} & -\mathrm{WBC}-1 \\
-\mathrm{C}^{-1} \mathrm{~B}^{T} \mathrm{~W} & \mathrm{C}^{-1}+\mathrm{C}^{-1} \mathrm{~B}^{T} \mathrm{WBC}^{-1}
\end{array}\right)
$$

where,

$$
\mathrm{W} \equiv\left(\mathrm{A}-\mathrm{BC} \mathrm{C}^{-1} \mathrm{~B}^{T}\right)^{-1}
$$

Subtracting the covariance for $\operatorname{Pr}\left(\Upsilon^{\prime} \mid a_{\mathrm{GP}}\right)$ from Equation $\mathrm{C} 4$ and adding the covariance $\Sigma(\theta)$ for $\operatorname{Pr}\left(\Upsilon^{\prime} \mid \theta\right)$ yields the covariance,

$$
\mathrm{M}^{-1} \equiv\left(\begin{array}{cc}
\mathrm{W} & -\mathrm{WBC}^{-1} \\
-\mathrm{C}^{-1} \mathrm{~B}^{T} \mathrm{~W} & \Sigma^{-1}(\theta)+\mathrm{C}^{-1} \mathrm{~B}^{T} \mathrm{WBC}^{-1}
\end{array}\right)
$$

Inverting gives the desired covariance matrix with a Gaussian cosmology prior imposed on the lens fields $\Upsilon\left(\mathbf{x}^{\prime}\right)$ at locations $\mathbf{x}^{\prime}$,

$$
\Sigma\left(\mathbf{x}, \mathbf{x}^{\prime} ; a_{\mathrm{GP}}, \theta\right)=\left(\begin{array}{cc}
\mathrm{A}-\mathrm{BC}^{-1} \mathrm{~B}^{T}+\mathrm{BC}^{-1} \Sigma(\theta) \mathrm{C}^{-1} \mathrm{~B}^{T} & \mathrm{BC}-1 \Sigma(\theta) \\
\Sigma(\theta) \mathrm{C}^{-1} \mathrm{~B}^{T} & \Sigma(\theta)
\end{array}\right) .
$$

As expected, Equation $\mathrm{C} 7$ reduces to $\Sigma\left(\mathbf{x}^{\prime} ; \theta\right)$ when the lens fields are evaluated only at the locations of the theory predictions $\mathbf{x}^{\prime}$. 